\title{
Success of extracorporeal shock wave lithotripsy and ERCP in symptomatic pancreatic duct stones: a systematic review and meta-analysis $\nabla$
}

\section{다)(1) $\odot(9$}

\author{
Authors \\ Nadine C.M. van Huijgevoort*, 1, Joyce V. Veld*, 1, Paul Fockens' ${ }^{1}$, Marc G. Besselink², Marja A. Boermeester ${ }^{2}$, \\ Marianna Arvanitakis ${ }^{3}$, Jeanin E. van Hooft ${ }^{1}$
}

Institutions

1 Department of Gastroenterology and Hepatology, Amsterdam Gastroenterology and Metabolism, Amsterdam UMC, location AMC, Amsterdam, The Netherlands

2 Department of Surgery, Amsterdam Gastroenterology and Metabolism, Amsterdam UMC, location AMC, Amsterdam, The Netherlands

3 Department of Gastroenterology and Hepatology, Erasme University Hospital, Université Libre de Bruxelles, Brussels, Belgium

submitted 19.12.2019

accepted after revision $\quad 30.3 .2020$

\author{
Bibliography \\ DOI https://doi.org/10.1055/a-1171-1322 | \\ Endoscopy International Open 2020; 08: E1070-E1085 \\ (c) Georg Thieme Verlag KG Stuttgart · New York \\ eISSN 2196-9736
}

Corresponding author

Jeanin E. van Hooft, MD, PhD, MBA, Amsterdam

Gastroenterology and Metabolism, Dept. of

Gastroenterology and Hepatology, C2-115, Amsterdam

UMC, location AMC, Meibergdreef 9, 1105 AZ Amsterdam,

The Netherlands

Fax: +31206917033

j.e.vanhooft@amsterdamumc.nl

$\circledast$ Supplementary material

Online content viewable at:

https://doi.org/10.1055/a-1171-1322
ABSTRACT

Background and study aims Pain is the most frequent and dominant symptom of chronic pancreatitis. Currently, these patients are treated using a step-up approach, including analgesics and lifestyle adjustments, endoscopic, and eventually surgical treatment. Extracorporeal shock wave lithotripsy (ESWL) is indicated after failure of the first step in patients with symptomatic intraductal stones larger than $5 \mathrm{~mm}$ in the head or body of the pancreas. To assess the complete ductal clearance rate and pain relief after ESWL in patients with symptomatic chronic pancreatitis with pancreatic duct stones, a systematic review and meta-analysis was performed.

Patients and methods A systematic literature search from January 2000 to December 2018 was performed in PubMed, the Cochrane Library, and EMBASE for studies on ductal clearance rate of ESWL in patients with symptomatic chronic pancreatitis with pancreatic duct stones.

Results After screening 486 studies, 22 studies with 3868 patients with chronic pancreatitis undergoing ESWL for pancreatic duct stones were included. The pooled proportion of patients with complete ductal clearance was $69.8 \%$ (95\% Cl 63.8-75.5). The pooled proportion of complete absence of pain during follow-up was $64.2 \%(95 \% \mathrm{Cl} 57.5-$ 70.6). Complete stone fragmentation was $86.3 \%(95 \% \mathrm{CI}$ 76.0-94.0). Post-procedural pancreatitis and cholangitis occurred in $4.0 \%(95 \% \mathrm{Cl} 2.5-5.8)$ and $0.5 \%(95 \% \mathrm{Cl} 0.2-$ $0.9)$, respectively.

Conclusion Treatment with ESWL results in complete ductal clearance rate in a majority of patients, resulting in absence of pain during follow up in over half of patients with symptomatic chronic pancreatitis caused by obstructing pancreatic duct stones.

\section{Introduction}

Chronic pancreatitis is an inflammatory condition of the pancreas, in which development of fibrosis and loss of pancreatic parenchyma may lead to impaired endocrine and exocrine pan-

\footnotetext{
* Drs. Van Huijgevoort and Veld: These authors contributed equally.
}

creatic function. Chronic pancreatitis is most frequently caused by alcohol and smoking, with idiopathic, genetic predisposition and autoimmunity as less frequent causes [1-3].

Pain is the most frequent and dominant symptom and has a highly variable clinical presentation, differing in chronicity and severity [4]. Pathogenesis of pain in chronic pancreatitis is mul- 
tifactorial, but poorly understood. Intraductal stones and/or strictures may lead to pancreatic ductal and pancreatic parenchymal hypertension, causing pain [5]. More recently, activation of intrapancreatic nociceptors, hypertrophy, and inflammation of intrapancreatic nerves and abnormal pain processing in the central nervous system have also been implicated [6].

For management of pain in chronic pancreatitis, a step-wise treatment is advocated, starting with lifestyle modifications, analgesics, plus, in selected patients, endoscopic therapy and/ or extracorporeal shock wave lithotripsy (ESWL) [7-9] with surgical decompression of the pancreatic duct as a last step (Supplementary Fig. 1). Endoscopic therapy and/or ESWL is indicated in patients with painful chronic pancreatitis with an obstructed main pancreatic duct (PD) in the head/body of the pancreas [8-10] (Supplementary Fig.2, Supplementary Fig. 3, - Video 1, > Video 2). Hypothetically, (endoscopic) PD drainage causes decompression of the duct, with a decrease in intraductal pressure and subsequent relief of pain as a result. For clearance of one or multiple radiopaque obstructive intraductal stones $\geq 5 \mathrm{~mm}$ located in the head/body of the pancreas, patients should be treated with ESWL, whereas endoscopic retrograde cholangiopancreatography (ERCP) is indicated for stones that are radiolucent or smaller than $5 \mathrm{~mm}$. Use of endoscopic therapy after ESWL has to be restricted to patients with PD strictures and no spontaneous clearance of the intraductal stones after adequate fragmentation by ESWL $[8,11]$. Clinical response to endoscopic therapy and/or ESWL should be evaluated 6 to 8 weeks after the procedure. If insufficient, surgical options have to be considered [9].

Recently, retrospective and prospective cohort studies have been published evaluating the efficacy and safety of ESWL in patients with symptomatic chronic pancreatitis. Therefore, the aim of this systematic review was to evaluate the efficacy of ductal clearance and pain relief from ESWL in treatment of patients with chronic pancreatitis with obstructing PD stones.

\section{Patients and methods}

\section{Study selection}

PRISMA guidelines reporting on meta-analyses and systematic reviews of observation studies were applied. A systematic literature search from January 2000 to December 2018 was performed in PubMed, the Cochrane Library, and EMBASE for studies on ductal clearance rate of ESWL in patients with symptomatic chronic pancreatitis with PD stones. Only full-text articles written in English were considered eligible. Search terms used were "lithotripsy," "extracorporeal shock wave lithotripsy," "stones," "pancreas," and "chronic pancreatitis" restricted to title, abstract, and keywords. Independent reviewers ( $\mathrm{NvH}$ and JV) screened each title and abstract for eligibility. Duplicate references were excluded. Discrepancies were solved through discussion and consensus, and in case of any doubt resolved with the senior author (JvH). Next, the eligibility of full text articles was assessed similarly. References of included articles were checked manually for studies that had not been identified in the primary search. Inclusion criteria were consecutive series of patients with chronic pancreatitis undergoing ESWL for

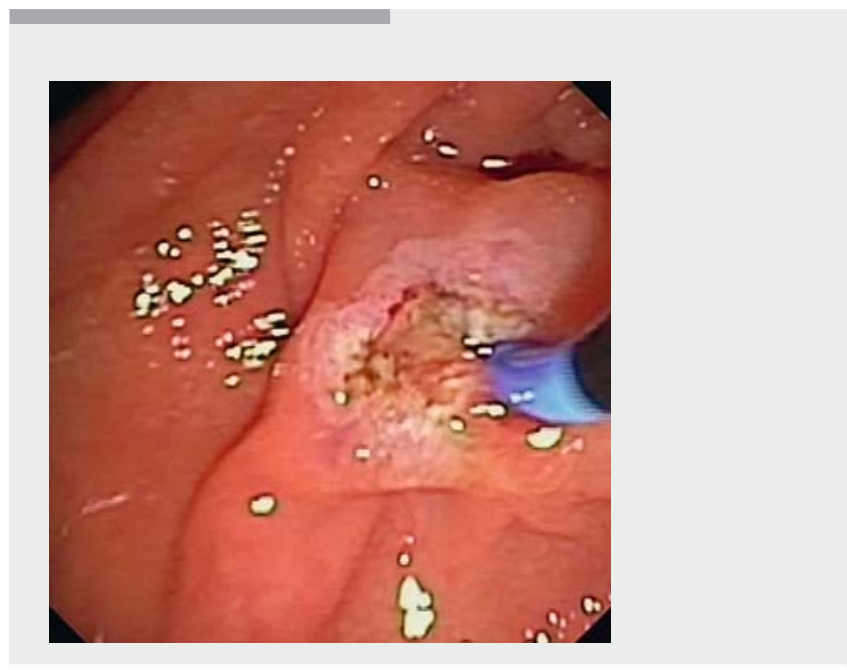

Video 1 A 50 year-old male patient with chronic calcifying pancreatitis and continuous pain had previously undergone ESWL with stone fragmentation. ERCP followed with pancreatic sphincterotomy and fragmented stone extraction is shown with a standard balloon catheter.

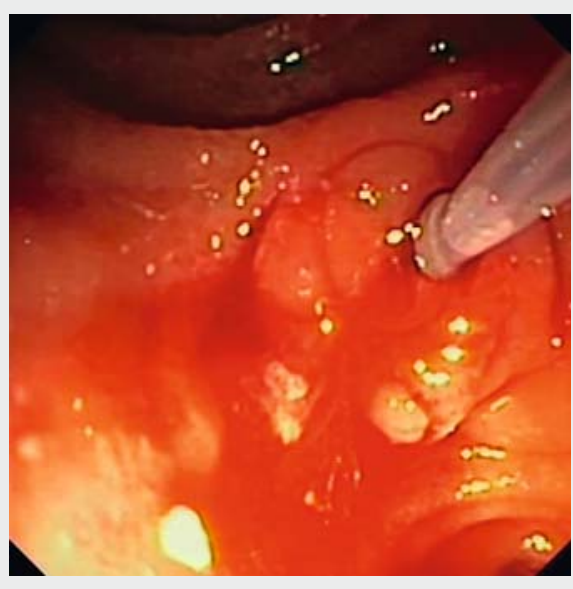

Video 2 A 60 year-old woman with chronic calcifying pancreatitis and continuous pain had previously undergone ESWL with partial stone fragmentation. The patient had already a previous pancreatic sphincterotomy and stone extraction was performed with a basket catheter, introduced along the guidewire, in the pancreatic duct. The catheter is flushed with saline during manipulation to facilitate fragment evacuation.

symptomatic PD stones, reporting of complete ductal clearance, and availability of a full article written in English. Exclusion criteria were studies with fewer than five patients and reviews, editorials, abstracts, letters, animal studies, or studies in children. The cut-off for minimum cohort size was arbitrarily chosen. 


\section{Data extraction}

The following variables were extracted, when available: study design, publication year, country of origin, number of patients undergoing ESWL, stone size (mean), complete stone fragmentation rate, complete stone fragmentation rate after first session, complete ductal clearance, complete ductal clearance after first session, number of session required for complete ductal clearance (mean), complications, follow-up time, pain relief, complete pain relief, partial pain relief, quality of life, exocrine insufficiency, and endocrine insufficiency.

\section{Outcomes}

Our primary outcome was complete ductal clearance rate. Complete ductal clearance was defined as the ability to retrieve at least $90 \%$ of all pancreatic duct stones with ESWL, including additional ERCP when applied. Secondary outcomes were patients being completely pain free after ESWL, complete ductal clearance after the first ESWL session, stone fragmentation rate, stone fragmentation rate after the first ESWL session, post-procedural pancreatitis rate, post-procedural cholangitis rate, and exocrine and endocrine insufficiency. Stone fragmentation was defined as the rupture of stones by ESWL.

\section{Methodological quality assessment}

All included studies were assessed for quality using The Oxford Centre for Evidence-Based Medicine Levels of Evidence checklist. The Newcastle-Ottawa Quality Assessment Scale for cohort studies was used to evaluate any risk of bias [12]. Any doubt regarding the methodological quality assessment was discussed by two independent reviewers ( $\mathrm{NvH}$ and JV).

\section{Statistical analysis}

Normally distributed variables were reported as means \pm standard deviation (SD), non-normally distributed variables as medians and interquartile range (IQR). Categorical data were reported as proportions. Heterogeneity among studies was assessed using the $\mathrm{I}^{2}$ statistic ( $\mathrm{I}^{2}$ value of $\geq 50 \%$ represented significant heterogeneity) [13]. Considering the variability of methods and populations in the included studies, a random effects model was used for analyses. Pooled proportions were calculated and presented as percentages with $95 \%$ confidence intervals $(95 \% \mathrm{Cl})$. Funnel plots of complete ductal clearance as our primary outcome were created to identify possible publication bias. Sensitivity analysis was performed by excluding retrospective studies and by excluding all studies in which postESWL ERCP was not performed. Analyses were performed with MedCalc version 18.5 (MedCalc Software).

\section{Results}

\section{Study selection}

The initial search contained 486 articles (Supplementary Fig. 4). After removal of 109 duplicates, 377 studies remained of which 342 were excluded based on title and abstract. After full text review, 22 eligible articles with 3868 patients undergoing ESWL for pancreatic duct stones were included. Cross-refer- ence did not reveal any studies missed with the initial search. No studies were excluded as a result of inadequate methodological quality (Supplemental Table1). An overview of study characteristics can be found in $>$ Table 1 .

\section{Complete ductal clearance}

All studies reported on complete ductal clearance ( $\downarrow$ Table 2 ). Meta-analysis of pooled proportions revealed complete ductal clearance in $69.8 \%(95 \% \mathrm{Cl} 63.8-75.5)$, as shown in $\mathbf{F i g . 1 a .}$ However, the test for heterogeneity did reveal a $P<0.001$ with a corresponding $\mathrm{I}^{2}$ of $92.6 \%$ ( $95 \% \mathrm{Cl} 90.1$ to 94.5$)$. Fig. 1 b represents publication bias for complete ductal clearance. As only two studies assessed complete ductal clearance after first ESWL session $[14,15]$, pooled analysis was not performed. Reported proportions of patients with complete ductal clearance after the first ESWL session were 60.2\% [14] and 68\% [15].

\section{Pain relief}

Pain intensity was heterogeneously reported among the respective studies ( $>$ Table 2 ). Pain intensity was measured using the Visal Analogue Scale (VAS) in four studies [14, 16-18]. The 3-point Likert-Scale was used in three studies to define pain intensity $[14,19,20]$. The 4 -grade Pain-Scale was used in two studies as a measure of pain intensity $[21,22]$. One of the authors [23] used the 5-point Likert-Scale to define pain intensity. In another study [24], a combination of the Visual Analogue Scale and the duration and frequency of the pain attacks was used to define pain intensity. One author [25] used the number of hospital admissions for pain during the follow-up period to define pain intensity. The proportion of patients being completely pain free following ESWL was reported in 6 studies $[16,17$, $19,20,23,24]$, showing a pooled proportion of $64.2 \%(95 \% \mathrm{Cl}$ 57.5-70.6) ( $\triangleright$ Fig. 2). The test for heterogeneity revealed $P=$ 0.014 with a corresponding $\mathrm{I}^{2}$ of $64.8 \%(95 \% \mathrm{Cl} 15.3-85.4)$. As the definition of partial pain relief was either highly heterogeneous or not defined amongst the respective studies, pooled proportion analysis was not performed on this outcome.

\section{Complete stone fragmentation}

A total of 12 studies including 1531 patients assessed complete stone fragmentation by ESWL $[15,18-20,22,24,26-31]$. The pooled proportion of patients in whom complete stone fragmentation was achieved was $86.3 \%$ (95\% Cl 76.0-94.0) as shown in $\mathbf{F i g . ~ 3 . ~ A s ~ w i t h ~ c o m p l e t e ~ d u c t a l ~ c l e a r a n c e , ~ t h e ~ s t u d - ~}$ ies showed a significant heterogeneity with $P<0.001$ and corresponding $\mathrm{I}^{2}$ of $95.9 \%(95 \% \mathrm{Cl} 94.2-97.1)$. As only three studies assessed complete fragmentation rate after first ESWL session $[18,19,32]$, pooled analysis was not performed regarding this outcome.

\section{Complications}

Pancreatitis as a complication following either ESWL or additional ERCP was reported in 15 studies $[14,15,18-20,23,25$, 26, 28, 29, 31-35] (Supplementary Table 2). In two studies it was unclear whether there was any overlap in patients with a post-ESWL and a post-ERCP pancreatitis, therefore these studies were excluded from the pooled proportion analysis [21, 22]. 
- Table 1 Study characteristics.

\begin{tabular}{|c|c|c|c|c|c|c|c|c|c|c|}
\hline & $\begin{array}{l}\text { Author, } \\
\text { year }\end{array}$ & $\begin{array}{l}\text { Coun- } \\
\text { try }\end{array}$ & $\begin{array}{l}\text { De- } \\
\text { sign }\end{array}$ & $n(m / f)$ & Age, y & $\begin{array}{l}\text { Pre- } \\
\text { ESWL } \\
\text { ERCP, } \\
\text { n/N } \\
\text { (\%) }\end{array}$ & $\begin{array}{l}\text { Location } \\
\text { stones, n/N } \\
\text { (\%) }\end{array}$ & $\begin{array}{l}\text { Stone size, } \\
\mathrm{mm}\end{array}$ & $\begin{array}{l}\text { MPD } \\
\text { stric- } \\
\text { tures, } \\
\text { n/N (\%) }\end{array}$ & ESWL equipment \\
\hline 1 & $\begin{array}{l}\text { Wang et } \\
\text { al. } 2018 \\
{[16]}\end{array}$ & China & $\mathrm{P}$ & $49(N R)^{1}$ & NR & NR & NR & NR & NR & $\begin{array}{l}\text { P-ESWL, third-genera- } \\
\text { tion electromagnetic li- } \\
\text { thotripter (Compact } \\
\text { Delta II; Dornier Med } \\
\text { Tech, Wessling, Germa- } \\
\text { ny) }\end{array}$ \\
\hline 2 & $\begin{array}{l}\text { Hu et al. } \\
2016[19]\end{array}$ & China & $\mathrm{P}$ & $\begin{array}{l}214 \\
(156 / \\
58)\end{array}$ & $\begin{array}{l}41.7 \pm \\
13.6\end{array}$ & $\begin{array}{l}128 / \\
214 \\
(60 \%)\end{array}$ & $\begin{array}{l}\text { Head: } 162 / 214 \\
(75.7 \%) \\
\text { Head/body: } 13 / \\
214(6.1 \%) \\
\text { Body: } 5 / 214 \\
(2.3 \%) \\
\text { Head/body/ } \\
\text { tail: } 34 / 214 \\
(15.9 \%)\end{array}$ & NR & NR & $\begin{array}{l}\text { Third-generation elec- } \\
\text { tromagnetic lithotripter } \\
\text { (Compact Delta II; Dor- } \\
\text { nier Med Tech, Wes- } \\
\text { sling, Germany) with } \\
\text { bidimensional fluoro- } \\
\text { scopic capability }\end{array}$ \\
\hline 3 & $\begin{array}{l}\text { Korpela et } \\
\text { al. } 2016 \\
{[14]}\end{array}$ & Finland & $\mathrm{R}$ & $\begin{array}{l}83(59 / \\
24)\end{array}$ & $\begin{array}{l}53(27- \\
77)^{2}\end{array}$ & $\begin{array}{l}74 / 83 \\
(89 \%)\end{array}$ & $\begin{array}{l}\text { Head: } 78 / 83 \\
(94.0 \%) \\
\text { Body: } 4 / 83 \\
(4.8 \%) \\
\text { Tail: } 1 / 83 \\
(1.2 \%)\end{array}$ & $10(5-25)^{2}$ & NR & $\begin{array}{l}\text { Electromagnetic litho- } \\
\text { tripter using fluoro- } \\
\text { scopic guidance. From } \\
\text { 2004-2005: Storz Mod- } \\
\text { ulith SLX, Medical AG, } \\
\text { Tägerwilen, Switzer- } \\
\text { land) From 2006-2013: } \\
\text { Modulith SLX-F2 }\end{array}$ \\
\hline 4 & $\begin{array}{l}\text { Lapp et al. } \\
2016 \text { [31] }\end{array}$ & U.S.A. & $R$ & $\begin{array}{l}37(21 / \\
16)\end{array}$ & $\begin{array}{l}57 \pm \\
13.7\end{array}$ & $\begin{array}{l}37 / 37 \\
(100 \%)\end{array}$ & $\begin{array}{l}\text { Head/neck: } 29 / \\
37(78.4 \%)\end{array}$ & $10.3 \pm 5.5$ & $\begin{array}{l}23 / 37 \\
(62.2 \%)\end{array}$ & $\begin{array}{l}\text { Piezolith } 3000 \text { (Knittlin- } \\
\text { gen, Germany) }\end{array}$ \\
\hline 5 & $\begin{array}{l}\text { Li et al. } \\
2016[20]\end{array}$ & China & $R$ & $\begin{array}{l}849 \\
(603 / \\
246) \\
\text { PPC: } 59 \\
(51 / 8) \\
\text { Non- } \\
\text { PPC: } 790 \\
(552 \mid \\
238)\end{array}$ & $\begin{array}{l}\text { PPC: } \\
41.6 \pm \\
13.3 \\
\text { Non- } \\
\text { PPC: } \\
41.0 \pm \\
14.2\end{array}$ & $\begin{array}{l}\text { PPC: } \\
14 / 59 \\
(23.7 \%) \\
\text { Non- } \\
\text { PPC: } \\
\text { NR }\end{array}$ & $\begin{array}{l}\text { PPC: } \\
\text { Head: } 14 / 59 \\
(23.7 \%) \\
\text { Tail: } 2 / 59 \\
(3.4 \%) \\
\text { Head \& at least } \\
\text { one other loca- } \\
\text { tion: } 43 / 59 \\
(72.3 \%)\end{array}$ & $\begin{array}{l}\text { PPC: } \\
5-10 \mathrm{~mm}: \\
22 / 59 \\
(37.3 \%) \\
10-20 \mathrm{~mm}: \\
29 / 59 \\
(49.2 \%) \\
20-30 \mathrm{~mm}: \\
6 / 59(10.2 \%) \\
\geq 30 \mathrm{~mm}: \\
2 / 59(3.4 \%)\end{array}$ & $\begin{array}{l}\text { PPC: } \\
40 / 59 \\
(67.8 \%)\end{array}$ & $\begin{array}{l}\text { P-ESWL, electromag- } \\
\text { netic lithotripter (Com- } \\
\text { pact Delta II; Dornier } \\
\text { Med Tech, Wessling, } \\
\text { Germany) }\end{array}$ \\
\hline 6 & $\begin{array}{l}\text { Vaysse et } \\
\text { al. } 2016 \\
{[35]}\end{array}$ & France & $P$ & $\begin{array}{l}146(96 / \\
50)\end{array}$ & $\begin{array}{l}51.2 \pm \\
13.4\end{array}$ & $\begin{array}{l}99 / 146 \\
(68 \%)\end{array}$ & NR & $\begin{array}{l}<10 \mathrm{~mm}: \\
29 / 132 \\
(22.0 \%) \\
\geq 10 \mathrm{~mm}: \\
37 / 132 \\
(28.0 \%) \\
\text { Missing: } 66 / \\
132(50.0 \%)\end{array}$ & NR & $\begin{array}{l}\text { Third-generation elec- } \\
\text { tromagnetic lithotripter } \\
\text { (Delta Compact; Dor- } \\
\text { nier Inc., Dornier Med- } \\
\text { tach, Munich, Germany) }\end{array}$ \\
\hline
\end{tabular}


- Table 1 (Continuation)

\begin{tabular}{|c|c|c|c|c|c|c|c|c|c|c|}
\hline & $\begin{array}{l}\text { Author, } \\
\text { year }\end{array}$ & $\begin{array}{l}\text { Coun- } \\
\text { try }\end{array}$ & $\begin{array}{l}\text { De- } \\
\text { sign }\end{array}$ & $n(m / f)$ & Age, y & $\begin{array}{l}\text { Pre- } \\
\text { ESWL } \\
\text { ERCP, } \\
\text { n/N } \\
(\%)\end{array}$ & $\begin{array}{l}\text { Location } \\
\text { stones, } \mathrm{n} / \mathrm{N} \\
\text { (\%) }\end{array}$ & $\begin{array}{l}\text { Stone size, } \\
\text { mm }\end{array}$ & $\begin{array}{l}\text { MPD } \\
\text { stric- } \\
\text { tures, } \\
\text { n/N (\%) }\end{array}$ & ESWL equipment \\
\hline 7 & $\begin{array}{l}\text { Maruya- } \\
\text { ma et al. } \\
2015[34]\end{array}$ & Japan & $\mathrm{R}$ & $\begin{array}{l}100(84 / \\
16) \\
\text { Chronic } \\
\text { AIP: } 8 \\
(7 / 1) \\
\text { Ordinary } \\
\text { CP: } 92 \\
(77 / 15)\end{array}$ & $\begin{array}{l}\text { Chronic } \\
\text { AIP: } 69 \\
(59-73)^{2} \\
\text { Ordinary } \\
\text { CP: } 56.5 \\
(20-85)^{2}\end{array}$ & $\begin{array}{l}100 / \\
100 \\
(100 \%)\end{array}$ & $\begin{array}{l}\text { Chronic AIP: } \\
\text { Head: } 6 / 8 \\
\text { (75.0\%) } \\
\text { Body: } 3 / 8 \\
(37.5 \%) \\
\text { Tail: 0/8 (0.0\%) } \\
\text { Ordinary CP: } \\
\text { Head: } 83 / 92 \\
(90.2 \%) \\
\text { Body: } 15 / 92 \\
(16.3 \%) \\
\text { Tail: } 1 / 92 \\
(1.1 \%)\end{array}$ & NR & NR & $\begin{array}{l}\text { Before 2004: Piezolith } \\
2500 \text { lithotriptor (Piezo- } \\
\text { electric effect tech- } \\
\text { nique; Richard Wolf } \\
\text { GmbH, Knittlingen, } \\
\text { Germany) } \\
\text { After 2004: LITHOSTAR } \\
\text { Multiline (Electromag- } \\
\text { netic generation tech- } \\
\text { nique; Siemens GmbH, } \\
\text { Munich, Germany) }\end{array}$ \\
\hline 8 & $\begin{array}{l}\text { Ohyama } \\
\text { et al. } 2015 \\
{[21]}\end{array}$ & Japan & $P$ & $\begin{array}{l}128(99 / \\
29)\end{array}$ & $\begin{array}{l}51.4 \pm \\
15.0\end{array}$ & $\begin{array}{l}128 / \\
128 \\
(100 \%)\end{array}$ & $\begin{array}{l}\text { Head: } 98 / 128 \\
\text { (76.6\%) } \\
\text { Body \& tail: } 30 / \\
128(23.4 \%)\end{array}$ & $12.2 \pm 5.5$ & $\begin{array}{l}73 / 128 \\
(57.0 \%)\end{array}$ & $\begin{array}{l}\text { Piezoelectric lithotrip- } \\
\text { ters (LT-01, } 02 \text { [EDAP In- } \\
\text { ternational Inc, Paris, } \\
\text { France]) or transporta- } \\
\text { ble cylindrical electro- } \\
\text { magnetic shock wave } \\
\text { generator device (Mod- } \\
\text { ulith SLZ; Storz Medical } \\
\text { AG, Kreuzlingen, Swit- } \\
\text { zerland) }\end{array}$ \\
\hline 9 & $\begin{array}{l}\text { Ito et al. } \\
2014 \text { [30] }\end{array}$ & Japan & $\mathrm{R}$ & $\begin{array}{l}98(78 / \\
20)\end{array}$ & $\begin{array}{l}54.8 \pm \\
13\end{array}$ & $\begin{array}{l}98 / 98 \\
(100 \%)\end{array}$ & $\begin{array}{l}\text { Single: } \\
\text { Head 18/24 } \\
(75 \%) \\
\text { Body/tail: 6/24 } \\
(25.0 \%) \\
\text { Multiple: } \\
\text { Head 58/74 } \\
(78.4 \%) \\
\text { Body/tail 16/74 } \\
(21.6 \%)\end{array}$ & $\begin{array}{l}>15: 11 / 98 \\
(11.2 \%) \\
\leq 15: 87 / 98 \\
(88.8 \%)\end{array}$ & $\begin{array}{l}62 / 98 \\
(63.3 \%)\end{array}$ & $\begin{array}{l}\text { Electromagnetic Sie- } \\
\text { mens Lithoskop (Sie- } \\
\text { mens AG, Munich, Ger- } \\
\text { many) }\end{array}$ \\
\hline 10 & $\begin{array}{l}\text { Suzuki et } \\
\text { al. } 2013 \\
{[29]}\end{array}$ & Japan & $R$ & 479 (NR) & NR & NR & NR & $\begin{array}{l}<10: 223 / \\
479(46.6 \%) \\
\geq 10,<2 \\
0: 162 / 479 \\
(33.8 \%) \\
\geq 20: 34 / 479 \\
(7.1 \%) \\
\text { Unknown } \\
60 / 479 \\
(12.5 \%)\end{array}$ & NR & $\begin{array}{l}\text { Electromagnetic system } \\
\mathrm{n}=290(60.5 \%) \\
\text { Electrohydraulic spark } \\
\text { gap system } \mathrm{n}=149 \\
(31.1 \%) \\
\text { Piezoelectric generator } \\
\mathrm{n}=22(4.6 \%) \\
\text { Unspecified } n=18 \\
(3.8 \%)\end{array}$ \\
\hline 11 & $\begin{array}{l}\text { Tandan et } \\
\text { al. } 2013 \\
{[17]}\end{array}$ & India & R & $\begin{array}{l}636 \\
(414 / \\
222)\end{array}$ & NR & NR & NR & NR & NR & $\begin{array}{l}\text { Third generation elec- } \\
\text { tromagnetic lithotripter } \\
\text { (Delta Compact, Dor- } \\
\text { nier Med Tech., Wes- } \\
\text { sling, Germany) }\end{array}$ \\
\hline 12 & $\begin{array}{l}\text { Merrill et } \\
\text { al. } 2011 \\
{[33]}\end{array}$ & USA & $\mathrm{R}$ & $\begin{array}{l}30(20 / \\
10)\end{array}$ & $\begin{array}{l}56.6(33- \\
71)^{3}\end{array}$ & $\begin{array}{l}30 / 30 \\
(100 \%)\end{array}$ & $\begin{array}{l}\text { Head: } 23 / 27 \\
(85.2 \%) \\
\text { Neck: } 1 / 27 \\
(3.7 \%) \\
\text { Body: } 2 / 27 \\
(7.4 \%) \\
\text { Tail: } 1 / 27 \\
(3.7 \%)\end{array}$ & $\begin{array}{l}<5=8 / 27 \\
(29.6 \%) \\
5-10=4 / 27 \\
(14.8 \%) \\
10-20= \\
15 / 27 \\
(55.6 \%)\end{array}$ & $\begin{array}{l}13 / 30 \\
(43.3 \%)\end{array}$ & $\begin{array}{l}\text { From 2002-2005: Dor- } \\
\text { nier HM3 (Medizintech- } \\
\text { nik GmbH, Germering, } \\
\text { Germany) From 2006- } \\
\text { 2009: Storz Modulith } \\
\text { SLX-F2 (Storz Medical } \\
\text { AG, Tagerwilen, Swit- } \\
\text { zerland) }\end{array}$ \\
\hline
\end{tabular}


- Table 1 (Continuation)

\begin{tabular}{|c|c|c|c|c|c|c|c|c|c|c|}
\hline & $\begin{array}{l}\text { Author, } \\
\text { year }\end{array}$ & $\begin{array}{l}\text { Coun- } \\
\text { try }\end{array}$ & $\begin{array}{l}\text { De- } \\
\text { sign }\end{array}$ & $n(m / f)$ & Age, y & $\begin{array}{l}\text { Pre- } \\
\text { ESWL } \\
\text { ERCP, } \\
\mathrm{n} / \mathrm{N} \\
(\%)\end{array}$ & $\begin{array}{l}\text { Location } \\
\text { stones, } \mathrm{n} / \mathrm{N} \\
(\%)\end{array}$ & $\begin{array}{l}\text { Stone size, } \\
\mathrm{mm}\end{array}$ & $\begin{array}{l}\text { MPD } \\
\text { stric- } \\
\text { tures, } \\
\text { n/N (\%) }\end{array}$ & ESWL equipment \\
\hline 13 & $\begin{array}{l}\text { Milovic et } \\
\text { al. } 2011 \\
{[23]}\end{array}$ & $\begin{array}{l}\text { Ger- } \\
\text { many }\end{array}$ & $\mathrm{P}$ & $\begin{array}{l}32(24 / \\
8)\end{array}$ & $\begin{array}{l}48(33- \\
76)^{2}\end{array}$ & $\begin{array}{l}32 / 32 \\
(100 \%)\end{array}$ & $\begin{array}{l}\text { Head: } 15 / 32 \\
(46.9 \%)\end{array}$ & NR & NR & $\begin{array}{l}\text { Modified mini-lithotrip- } \\
\text { ter (Minilith SL 1, Storz, } \\
\text { Switzerland) }\end{array}$ \\
\hline 14 & $\begin{array}{l}\text { Parsi et al. } \\
2010 \text { [32] }\end{array}$ & USA & $\mathrm{P}$ & $10(3 / 7)$ & $\begin{array}{l}54(44- \\
72)^{3}\end{array}$ & $\begin{array}{l}10 / 10 \\
(100 \%)\end{array}$ & $\begin{array}{l}\text { Head: } 4 / 10 \\
(40 \%) \\
\text { Head/body: } \\
2 / 10(20 \%) \\
\text { Head/body/ } \\
\text { tail: } 4 / 10(40 \%)\end{array}$ & $15(10-20)^{3}$ & NR & $\begin{array}{l}\text { Transportable electro- } \\
\text { magnetic shock wave } \\
\text { generator device (Mod- } \\
\text { ulith SLX-2; Storz Medi- } \\
\text { cal AG, Kreuzlingen, } \\
\text { Switzerland) }\end{array}$ \\
\hline 15 & $\begin{array}{l}\text { Tadenu- } \\
\text { ma et al. } \\
2005 \text { [22] }\end{array}$ & Japan & $\mathrm{R}$ & $\begin{array}{l}117(85 / \\
32)\end{array}$ & $\begin{array}{l}48(12- \\
73)^{3}\end{array}$ & $\begin{array}{l}117 / \\
117 \\
(100 \%)\end{array}$ & $\begin{array}{l}\text { Head: } 100 / 117 \\
(85.5 \%) \\
\text { Body/tail: } 17 / \\
117(14.5 \%)\end{array}$ & $11.2(3-37)^{3}$ & $\begin{array}{l}57 / 117 \\
(48.7 \%)\end{array}$ & $\begin{array}{l}\text { Piezoelectric lithotripter } \\
\text { (LT-01, 02; EDAP Inter- } \\
\text { national Inc., Paris, } \\
\text { France) or Piezolith } \\
\text { 2500; Richard Wolf, Inc., } \\
\text { Knittlingen, Germany) }\end{array}$ \\
\hline 16 & $\begin{array}{l}\text { Inui et al. } \\
2005 \text { [28] }\end{array}$ & Japan & $\mathrm{R}$ & $\begin{array}{l}555 \\
(465 / \\
90)\end{array}$ & $\begin{array}{l}52.5(12- \\
79)^{3}\end{array}$ & NR & NR & NR & NR & $\begin{array}{l}\text { Electrohydraulic spark } \\
\text { gap system } n=167 \\
\text { Piezoelectric generator } \\
\mathrm{n}=109 \\
\text { Electrohydraulic spark } \\
\text { gap/electromagnetic } \\
\text { system } \mathrm{n}=74 \\
\text { Electromagnetic system } \\
\mathrm{n}=205\end{array}$ \\
\hline 17 & $\begin{array}{l}\text { Delhaye } \\
\text { et al. } 2004 \\
\text { [25] }\end{array}$ & $\begin{array}{l}\text { Bel- } \\
\text { gium }\end{array}$ & $\mathrm{R}$ & $\begin{array}{l}56(46 / \\
10)\end{array}$ & $\begin{array}{l}44 \pm \\
12.5\end{array}$ & $\begin{array}{l}56 / 56 \\
(100 \%)\end{array}$ & $\begin{array}{l}\text { Distal: } 44 / 56 \\
\text { (78.6\%) } \\
\text { Non-distal: } \\
\text { 5/56 (8.9\%) } \\
\text { Both: } 7 / 56 \\
(12.5 \%)\end{array}$ & $13 \pm 5.9$ & $\begin{array}{l}35 / 56 \\
(62 \%)\end{array}$ & $\begin{array}{l}\text { Electromagnetic litho- } \\
\text { tripter (Siemens Lithos- } \\
\text { tar, Erlangen, Germany) }\end{array}$ \\
\hline 18 & $\begin{array}{l}\text { Farnba- } \\
\text { cher et al. } \\
2002 \text { [27] }\end{array}$ & $\begin{array}{l}\text { Ger- } \\
\text { many }\end{array}$ & $\mathrm{R}$ & 114 (NR) & NR & $\begin{array}{l}114 / \\
114 \\
(100 \%)\end{array}$ & NR & NR & NR & $\begin{array}{l}\text { Piezoelectric shockwave } \\
\text { lithotripter with cap- } \\
\text { ability for visualization } \\
\text { ultrasonographically } \\
\text { (Piezolith } 2300, \text { R. Wolf, } \\
\text { Knittlingen, Germany) } \\
\text { or with alternating ul- } \\
\text { trasound and radio- } \\
\text { graphic visualization } \\
\text { (Piezolith 2500, Piezo- } \\
\text { lith 2501-economy; } \\
\text { Wolf) }\end{array}$ \\
\hline 19 & $\begin{array}{l}\text { Kozarek et } \\
\text { al. } 2002 \\
{[18]}\end{array}$ & USA & $\mathrm{R}$ & $\begin{array}{l}40(21 / \\
19)\end{array}$ & $\begin{array}{l}53(23- \\
77)^{3}\end{array}$ & $\begin{array}{l}40 / 40 \\
(100 \%)\end{array}$ & NR & $13(4-25)^{3}$ & $\begin{array}{l}19 / 40 \\
(47.5 \%)\end{array}$ & $\begin{array}{l}\text { Dornier HM3 lithotriptor } \\
\text { (Dornier, Inc., Dornier } \\
\text { Medtech, Munich, Ger- } \\
\text { many) }\end{array}$ \\
\hline 20 & $\begin{array}{l}\text { Ruben- } \\
\text { stein et al. } \\
2002 \text { [15] }\end{array}$ & USA & $\mathrm{P}$ & $\begin{array}{l}23(11 / \\
12) \\
\text { HM3: } 11 \\
(3 / 8) \\
\text { Litho- } \\
\text { tron: } 12 \\
(9 / 3)\end{array}$ & $\begin{array}{l}52.1 \\
\text { (NR) } \\
\text { HM3: } \\
52.5 \\
\text { (NR) } \\
\text { Litho- } \\
\text { tron: } \\
51.7 \\
\text { (NR) }\end{array}$ & $\begin{array}{l}23 / 23 \\
(100 \%)\end{array}$ & NR & $\begin{array}{l}17 \text { (NR) } \\
\text { HM3: } 21 \\
\text { (NR) } \\
\text { Lithotron: } 14 \\
\text { (NR) }\end{array}$ & $\begin{array}{l}13 / 23 \\
(56.5 \%) \\
\text { HM3: } \\
6 / 11 \\
(54.5 \%) \\
\text { Litho- } \\
\text { tron: } \\
7 / 12 \\
(58.3 \%)\end{array}$ & $\begin{array}{l}\text { HM3 lithotriptor or Li- } \\
\text { thotron lithotriptor }\end{array}$ \\
\hline
\end{tabular}




\begin{tabular}{|c|c|c|c|c|c|c|c|c|c|c|}
\hline & $\begin{array}{l}\text { Author, } \\
\text { year }\end{array}$ & $\begin{array}{l}\text { Coun- } \\
\text { try }\end{array}$ & $\begin{array}{l}\text { De- } \\
\text { sign }\end{array}$ & $n(m / f)$ & Age, y & $\begin{array}{l}\text { Pre- } \\
\text { ESWL } \\
\text { ERCP, } \\
\text { n/N } \\
(\%)\end{array}$ & $\begin{array}{l}\text { Location } \\
\text { stones, } \mathrm{n} / \mathrm{N} \\
\text { (\%) }\end{array}$ & $\begin{array}{l}\text { Stone size, } \\
\text { mm }\end{array}$ & $\begin{array}{l}\text { MPD } \\
\text { stric- } \\
\text { tures, } \\
\mathrm{n} / \mathrm{N}(\%)\end{array}$ & ESWL equipment \\
\hline 21 & $\begin{array}{l}\text { Karasawa } \\
\text { et al. } 2002 \\
{[26]}\end{array}$ & Japan & $\mathrm{R}$ & $\begin{array}{l}24(19 / \\
5)\end{array}$ & $53 \pm 18$ & $\begin{array}{l}24 / 24 \\
(100 \%)\end{array}$ & $\begin{array}{l}\text { Head: } 11 / 24 \\
(45.8 \%) \\
\text { Head/body/ } \\
\text { tail: } 13 / 24 \\
(54.2 \%)\end{array}$ & $10.6 \pm 3.7$ & $\begin{array}{l}12 / 24 \\
(50 \%)\end{array}$ & $\begin{array}{l}\text { Piezoelectric lithotripter } \\
\text { (Piezolith 2500; Richard } \\
\text { Wolf, Knittlingen, Ger- } \\
\text { many) }\end{array}$ \\
\hline 22 & $\begin{array}{l}\text { Brand et } \\
\text { al. } 2000 \\
{[24]}\end{array}$ & $\begin{array}{l}\text { Ger- } \\
\text { many }\end{array}$ & $P$ & $\begin{array}{l}48(35 / \\
13)\end{array}$ & $\begin{array}{l}51(14- \\
74)^{3}\end{array}$ & $\begin{array}{l}44 / 48 \\
(92 \%)\end{array}$ & $\begin{array}{l}\text { Head: } 26 / 48 \\
(54.2 \%) \\
\text { Body: } 10 / 48 \\
(20.8 \%) \\
\text { Tail: } 12 / 48 \\
(25 \%)\end{array}$ & $12(4-30)^{2}$ & $\begin{array}{l}34 / 48 \\
(71 \%)\end{array}$ & $\begin{array}{l}\text { Electromagnetic shock- } \\
\text { wave lithotripter (modi- } \\
\text { fied Lithostar prototype, } \\
\text { Siemens, Erlangen, Ger- } \\
\text { many) }\end{array}$ \\
\hline \multicolumn{11}{|c|}{$\begin{array}{l}\text { AIP, autoimmune pancreatitis; } C P \text {, chronic pa } \\
f \text {, female; NR, not reported; } P \text {, prospective; } M \\
\text { y, year } \\
{ }^{1} \text { Only matched controls included in analysis } \\
{ }^{2} \text { Median with range } \\
{ }^{3} \text { Mean with range }\end{array}$} \\
\hline
\end{tabular}

The pooled proportion of pancreatitis was $4.0 \%(95 \% \mathrm{Cl} 2.5-$ 5.8) (Supplementary Fig. 5). The studies showed a significant heterogeneity with $P<0.001$ and corresponding $\mathrm{I}^{2}$ of $69.1 \%$ ( $95 \% \mathrm{Cl} 47.5-81.9)$. A total of 16 studies reported on cholangitis as a complication $[14,15,18-23,26,28,29,31,32,34,35]$. The pooled proportion of cholangitis was $0.5 \%(95 \% \mathrm{Cl} 0.2-$ 0.9) (Supplementary Fig.6). The studies did not show significant heterogeneity with a P-value of 0.055 and corresponding $I^{2}$ of $39.1 \%$ (95\% Cl 0.0-66.4).

\section{Exocrine deficiency}

In total, 10 studies reported on exocrine function following ESWL treatment $[14,17,19-22,24-26,28]$ ( $\bullet$ Table 3). Four studies measured exocrine function following ESWL using the $\mathrm{N}$-benzoyl-I-tyrosyl-para-aminobenzoic acid (BT-PABA) test $[21,22,26,28]$. Two studies used fecal elastase to define exocrine deficiency [14, 24]; one of them [14] only measured fecal elastase- 1 in case of self-reported diarrhea or steatorrhea. Fecal chymotrypsin was used in two studies as a measure of exocrine deficiency $[24,26]$. Two studies $[19,24]$ used self-reported steatorrhea as a measure for exocrine deficiency, whereas a third study [17] combined this self-reported measure with the amount of pancreatic enzymes required to relieve symptoms. Another study [25] defined exocrine insufficiency as clinical steatorrhea that improved with more than 1 month of treatment with pancreatic enzyme substitution. In the final study [20], it was unknown how exocrine deficiency was defined.

\section{Endocrine deficiency}

In total, 10 studies reported on endocrine function following ESWL treatment $[14,17,19-22,24-26,28]$ ( $\triangleright$ Table 3$)$. One study [26] reported on patients requiring antidiabetic treat- ment after ESWL. Another [25] reported on patients requiring oral antidiabetic drugs or insulin for more than one month. Two of the studies $[19,14]$ used self-reported diabetes as a measure for endocrine deficiency. Three $[17,24,28]$ measured blood glucose, HbA1c, and the required dose of hypoglycemic agents to define endocrine deficiency. Another study [24] further defined endocrine deficiency as a fasting blood glucose level $>126 \mathrm{mg} / \mathrm{dL}$ and an $\mathrm{HbA} 1 \mathrm{c}>6.0 \%$. One author [20] defined endocrine insufficiency as 'patients with diabetes following ESWL, whereas two other authors [21,22] additionally referred to the American Diabetes Association.

\section{Quality of life}

Quality of life was heterogeneously reported among the respective studies $[14,17,19,20,23,24]$, therefore, no pooled analysis could be performed for this outcome. Three of the studies used a scale ranging from $1-10[17,19,20]$, whereas the two others used a 1-5 scale [23] and a European Organization for Research and Treatment of Cancer (EORTC-QLQ) questionnaire with a scale ranging from $0-100$ [24], respectively. One study [14] did not report a numeric score but a description of daily activities obtained through telephonic interviews.

\section{Sensitivity analysis}

Sensitivity analysis was performed after excluding all studies in which post-ESWL ERCP was not performed ( $\bullet$ Table 1$)$ ). A total of 12 studies with 1284 (33.2\%) patients were included in the sensitivity analysis. Complete ductal clearance was reached in 71.4\% (95\% Cl 61.3-80.5) patients undergoing ESWL with ERCP. Complete pain relief was described in three of the 12 studies with 706 patients. The proportion of patients being completely pain free at follow up after ESWL with ERCP was 
- Table 2 Outcomes after ESWL.

\begin{tabular}{|c|c|c|c|c|c|c|c|c|c|c|c|}
\hline & $\begin{array}{l}\text { Author, } \\
\text { year }\end{array}$ & $\begin{array}{l}\text { Complete } \\
\text { stone frag- } \\
\text { mentation, } \\
\text { n/N (\%) }\end{array}$ & $\begin{array}{l}\text { Complete } \\
\text { stone frag- } \\
\text { mentation } \\
\text { after first } \\
\text { session, } \\
\text { n/N (\%) }\end{array}$ & $\begin{array}{l}\text { Com- } \\
\text { plete } \\
\text { ductal } \\
\text { clear- } \\
\text { ance } \\
\text { after } \\
\text { first } \\
\text { session, } \\
\text { n/N (\%) }\end{array}$ & $\begin{array}{l}\text { Post- } \\
\text { ESWL } \\
\text { ERCP, } \\
\text { n/N (\%) }\end{array}$ & $\begin{array}{l}\text { Com- } \\
\text { plete } \\
\text { ductal } \\
\text { clear- } \\
\text { ance, } \\
\mathrm{n} / \mathrm{N}(\%)\end{array}$ & $\begin{array}{l}\text { Method of } \\
\text { pain } \\
\text { measure- } \\
\text { ment }\end{array}$ & $\begin{array}{l}\text { Pain } \\
\text { relief, } \\
\text { n/N (\%) }\end{array}$ & $\begin{array}{l}\text { Com- } \\
\text { plete } \\
\text { pain } \\
\text { relief, } \\
\text { n/N (\%) }\end{array}$ & $\begin{array}{l}\text { Par- } \\
\text { tial } \\
\text { pain } \\
\text { relief, } \\
\mathrm{n} / \mathrm{N} \\
(\%)\end{array}$ & $\begin{array}{l}\text { Follow- } \\
\text { up, m }\end{array}$ \\
\hline 1 & $\begin{array}{l}\text { Wang } \\
\text { et al. } \\
2018 \\
{[16]^{1}}\end{array}$ & NR & NR & NR & NR & $\begin{array}{l}44 / 49 \\
(89.8 \%)\end{array}$ & VAS-score & $\begin{array}{l}44 / 49 \\
(89.8 \%)\end{array}$ & $\begin{array}{l}37 / 49 \\
(75.5 \%)\end{array}$ & $\begin{array}{l}7 / 49 \\
(14.3 \%)\end{array}$ & NR \\
\hline 2 & $\begin{array}{l}\text { Hu et al. } \\
2016 \\
{[19]}\end{array}$ & $\begin{array}{l}214 / 214 \\
(100 \%)\end{array}$ & $\begin{array}{l}101 / 214 \\
(47.2 \%)\end{array}$ & NR & $\begin{array}{l}207 / \\
214 \\
(96.7 \%)\end{array}$ & $\begin{array}{l}155 / 214 \\
(72.4 \%)\end{array}$ & $\begin{array}{l}\text { 3-point } \\
\text { Likert scale }\end{array}$ & $\begin{array}{l}186 / 195 \\
(95.4 \%)\end{array}$ & $\begin{array}{l}139 / \\
195 \\
(71.3 \%)\end{array}$ & $\begin{array}{l}47 / 195 \\
(24.1 \%)\end{array}$ & $\begin{array}{l}18.5 \pm 3.3 \\
(n=195)\end{array}$ \\
\hline 3 & $\begin{array}{l}\text { Korpela } \\
\text { et al. } \\
2016 \\
{[14]}\end{array}$ & NR & NR & $\begin{array}{l}50 / 83 \\
(60.2 \%)\end{array}$ & $\begin{array}{l}83 / 83 \\
(100 \%) \\
28 / 83 \\
(100 \%) \\
\text { (same } \\
\text { day as } \\
\text { ESWL) }\end{array}$ & $\begin{array}{l}69 / 83 \\
(83.1 \%)\end{array}$ & $\begin{array}{l}\text { VAS-score } \\
\text { 3-point } \\
\text { Likert scale }\end{array}$ & $\begin{array}{l}74 / 83 \\
(89.2 \%)\end{array}$ & NR & NR & $\begin{array}{l}12(1-36)^{2} \\
(n=83)\end{array}$ \\
\hline 4 & $\begin{array}{l}\text { Lapp et } \\
\text { al. } 2016 \\
{[31]}\end{array}$ & $\begin{array}{l}22 / 37 \\
(59.5 \%)\end{array}$ & NR & NR & $\begin{array}{l}36 / 37 \\
(97.3 \%)\end{array}$ & $\begin{array}{l}29 / 36 \\
(80.6 \%)\end{array}$ & NR & $\begin{array}{l}14 / 37 \\
(37.8 \%)\end{array}$ & NR & NR & NR \\
\hline 5 & $\begin{array}{l}\text { Li et al. } \\
2016 \\
{[20]}\end{array}$ & $\begin{array}{l}\text { PPC: } 59 / 59 \\
(100 \%) \\
\text { Non-PPC: } \\
\text { NR }\end{array}$ & NR & NR & $\begin{array}{l}\text { PPC: } \\
58 / 59 \\
(98.3 \%) \\
\text { Non- } \\
\text { PPC: NR }\end{array}$ & $\begin{array}{l}696 / 849 \\
(82.0 \%) \\
\text { PPC: 39/ } \\
58 \\
(67.2 \%) \\
\text { Non-PPC: } \\
657 / 790 \\
(83.2 \%)\end{array}$ & $\begin{array}{l}\text { 3-point } \\
\text { Likert scale }\end{array}$ & $\begin{array}{l}\text { PPC: } 49 / \\
55 \\
(89.1 \%)\end{array}$ & $\begin{array}{l}\text { PPC: } \\
35 / 55 \\
(63.6 \%)\end{array}$ & $\begin{array}{l}\text { PPC: } \\
14 / 55 \\
(25.5 \%)\end{array}$ & $\begin{array}{l}21.9 \\
(12.0- \\
45.1)^{2} \\
(n=55 ; \\
\text { PPC } \\
\text { group) }\end{array}$ \\
\hline 6 & $\begin{array}{l}\text { Vaysse } \\
\text { et al. } \\
2016 \\
{[35]}\end{array}$ & NR & NR & NR & $\begin{array}{l}91 / 132 \\
(73.5 \%)\end{array}$ & $\begin{array}{l}75 / 132 \\
(56.8 \%)\end{array}$ & NR & NR & NR & NR & $\begin{array}{l}23(6-90)^{2} \\
(n=132 \\
\text { with 6- } \\
\text { month FU) }\end{array}$ \\
\hline 7 & $\begin{array}{l}\text { Mar- } \\
\text { uyama } \\
\text { et al. } \\
2015 \\
{[34]}\end{array}$ & NR & NR & NR & $\begin{array}{l}100 / \\
100 \\
(100 \%)\end{array}$ & $\begin{array}{l}76 / 100 \\
(76.0 \%) \\
\text { Chronic } \\
\text { AIP: } \\
5 / 8 \\
(62.5 \%) \\
\text { Ordinary } \\
\text { CP: } \\
71 / 92 \\
(77.2 \%)\end{array}$ & NR & NR & NR & NR & $\begin{array}{l}68(36- \\
180)^{2} \\
(n=8 ; \\
\text { chronic } \\
\text { AIP) }\end{array}$ \\
\hline 8 & $\begin{array}{l}\text { Ohya- } \\
\text { ma et } \\
\text { al. } 2015 \\
\text { [21] }\end{array}$ & NR & NR & NR & $\begin{array}{l}128 / \\
128 \\
(100 \%)\end{array}$ & $\begin{array}{l}66 / 128 \\
(51.6 \%)\end{array}$ & $\begin{array}{l}\text { 4-grade } \\
\text { pain scale }\end{array}$ & $\begin{array}{l}115 / 128 \\
(89.9 \%)\end{array}$ & NR & NR & $\begin{array}{l}42.4 \pm \\
35.8 \\
(n=N R)\end{array}$ \\
\hline 9 & $\begin{array}{l}\text { Ito et al. } \\
2014 \\
{[30]}\end{array}$ & $\begin{array}{l}67 / 98 \\
(100 \%)\end{array}$ & NR & NR & $\begin{array}{l}98 / 98 \\
(100 \%)\end{array}$ & $\begin{array}{l}72 / 98 \\
(73.5 \%)\end{array}$ & NR & $\begin{array}{l}81 / 89 \\
(91.0 \%)\end{array}$ & NR & NR & NR \\
\hline 10 & $\begin{array}{l}\text { Suzuki } \\
\text { et al. } \\
2013 \\
{[29]}\end{array}$ & $\begin{array}{l}186 / 202 \\
(92.1 \%)^{3}\end{array}$ & NR & NR & $\begin{array}{l}255 / \\
479 \\
(53.2 \%)\end{array}$ & $\begin{array}{l}356 / 479 \\
(74.3 \%)\end{array}$ & NR & $\begin{array}{l}435 / 479 \\
(90.8 \%)\end{array}$ & NR & NR & $\begin{array}{l}31.4 \\
(1-83)^{4} \\
(n=479)\end{array}$ \\
\hline
\end{tabular}




\begin{tabular}{|c|c|c|c|c|c|c|c|c|c|c|c|}
\hline & $\begin{array}{l}\text { Author, } \\
\text { year }\end{array}$ & $\begin{array}{l}\text { Complete } \\
\text { stone frag- } \\
\text { mentation, } \\
\text { n/N (\%) }\end{array}$ & $\begin{array}{l}\text { Complete } \\
\text { stone frag- } \\
\text { mentation } \\
\text { after first } \\
\text { session, } \\
\text { n/N (\%) }\end{array}$ & $\begin{array}{l}\text { Com- } \\
\text { plete } \\
\text { ductal } \\
\text { clear- } \\
\text { ance } \\
\text { after } \\
\text { first } \\
\text { session, } \\
\text { n/N (\%) }\end{array}$ & $\begin{array}{l}\text { Post- } \\
\text { ESWL } \\
\text { ERCP, } \\
\text { n/N (\%) }\end{array}$ & $\begin{array}{l}\text { Com- } \\
\text { plete } \\
\text { ductal } \\
\text { clear- } \\
\text { ance, } \\
\text { n/N (\%) }\end{array}$ & $\begin{array}{l}\text { Method of } \\
\text { pain } \\
\text { measure- } \\
\text { ment }\end{array}$ & $\begin{array}{l}\text { Pain } \\
\text { relief, } \\
\mathrm{n} / \mathrm{N}(\%)\end{array}$ & $\begin{array}{l}\text { Com- } \\
\text { plete } \\
\text { pain } \\
\text { relief, } \\
\text { n/N (\%) }\end{array}$ & $\begin{array}{l}\text { Par- } \\
\text { tial } \\
\text { pain } \\
\text { relief, } \\
\mathrm{n} / \mathrm{N} \\
(\%)\end{array}$ & $\begin{array}{l}\text { Follow- } \\
\text { up, } m\end{array}$ \\
\hline 11 & $\begin{array}{l}\text { Tandan } \\
\text { et al. } \\
2013 \\
{[17]}\end{array}$ & NR & NR & NR & $\begin{array}{l}636 / \\
636 \\
(100 \%)\end{array}$ & $\begin{array}{l}489 / 636 \\
(76.9 \%) \\
282 / 364 \\
(77.5 \%)^{5} \\
207 / 272 \\
(76.1 \%)^{7}\end{array}$ & VAS-score & NR & $\begin{array}{l}414 \mid \\
636 \\
(65.1 \%) \\
250 / \\
364 \\
(68.7 \%) \\
5 \\
164 \mid \\
272 \\
(60.3 \%) \\
6\end{array}$ & NR & $\begin{array}{l}\text { Inter- } \\
\text { mediate } \\
\text { follow-up } \\
24-60 \\
(n=364) \\
\text { Long- } \\
\text { term fol- } \\
\text { low-up } \\
>60(n= \\
272)\end{array}$ \\
\hline 12 & $\begin{array}{l}\text { Merrill } \\
\text { et al. } \\
2011 \\
{[33]}\end{array}$ & NR & NR & NR & $\begin{array}{l}30 / 30 \\
(100 \%)\end{array}$ & $\begin{array}{l}27 / 30 \\
(90.0 \%) \\
12 / 30 \\
(40.0 \%)^{7}\end{array}$ & NR & NR & NR & NR & NR \\
\hline 13 & $\begin{array}{l}\text { Milovic } \\
\text { et al. } \\
2011 \\
{[23]}\end{array}$ & NR & NR & NR & $\begin{array}{l}32 / 32 \\
(100 \%)\end{array}$ & $\begin{array}{l}13 / 32 \\
(40.6 \%)\end{array}$ & $\begin{array}{l}\text { 5-point } \\
\text { Likert scale }\end{array}$ & $\begin{array}{l}28 / 32 \\
(87.5 \%) \\
24 / 32 \\
(75.0 \%)^{8}\end{array}$ & $\begin{array}{l}17 / 32 \\
(53.1 \%) \\
7 / 32 \\
(21.9 \%)^{8}\end{array}$ & NR & NR \\
\hline 14 & $\begin{array}{l}\text { Parsi et } \\
\text { al. } 2010 \\
{[32]}\end{array}$ & NR & $\begin{array}{l}8 / 10 \\
(80.0 \%)\end{array}$ & NR & $\begin{array}{l}10 / 10 \\
(100 \%)\end{array}$ & $\begin{array}{l}7 / 10 \\
(70.0 \%)\end{array}$ & NR & NR & NR & NR & $\begin{array}{l}20(12- \\
36)^{4} \\
(n=10)\end{array}$ \\
\hline 15 & $\begin{array}{l}\text { Tade- } \\
\text { numa et } \\
\text { al. } 2005 \\
{[22]}\end{array}$ & $\begin{array}{l}113 / 117 \\
(96.6 \%)\end{array}$ & NR & NR & $\begin{array}{l}65 / 117 \\
(55.6 \%)\end{array}$ & $\begin{array}{l}65 / 117 \\
(55.6 \%)\end{array}$ & $\begin{array}{l}\text { 4-grade } \\
\text { pain scale }\end{array}$ & $\begin{array}{l}49 / 70 \\
(70.0 \%) \\
114 / 117 \\
(68.2 \%)^{8}\end{array}$ & NR & NR & $\begin{array}{l}77.5 \pm \\
30.9 \\
(n=70)\end{array}$ \\
\hline 16 & $\begin{array}{l}\text { Inui et } \\
\text { al. } 2005 \\
{[28]}\end{array}$ & $\begin{array}{l}513 / 555 \\
(92.4 \%)\end{array}$ & NR & NR & $\begin{array}{l}237 \mid \\
555 \\
(42.7 \%)\end{array}$ & $\begin{array}{l}403 / 555 \\
(72.6 \%)\end{array}$ & NR & $\begin{array}{l}428 / 470 \\
(91.1 \%)\end{array}$ & NR & $\begin{array}{l}428 / \\
470 \\
(91.1 \%)\end{array}$ & $\begin{array}{l}44.3 \\
(3-141)^{4} \\
(n=504)\end{array}$ \\
\hline 17 & $\begin{array}{l}\text { Delhaye } \\
\text { et al. } \\
2004 \\
{[25]}\end{array}$ & NR & NR & NR & $\begin{array}{l}56 / 56 \\
(100 \%)\end{array}$ & $\begin{array}{l}27 / 56 \\
(48 \%)\end{array}$ & $\begin{array}{l}\text { Number of } \\
\text { hospital } \\
\text { admis- } \\
\text { sions for } \\
\text { pain dur- } \\
\text { ing the fol- } \\
\text { low-up } \\
\text { period }\end{array}$ & NR & NR & NR & $\begin{array}{l}14.4 \pm 0.6 \\
\text { years }\end{array}$ \\
\hline 18 & $\begin{array}{l}\text { Farnba- } \\
\text { cher et } \\
\text { al. } 2002 \\
{[27]}\end{array}$ & $\begin{array}{l}94 / 114 \\
(82.5 \%)\end{array}$ & NR & NR & NR & $\begin{array}{l}39 / 114 \\
(34.2 \%)\end{array}$ & NR & NR & NR & NR & NR \\
\hline 19 & $\begin{array}{l}\text { Kozarek } \\
\text { et al. } \\
2002 \\
{[18]}\end{array}$ & $\begin{array}{l}40 / 40 \\
(100 \%)\end{array}$ & $\begin{array}{l}35 / 40 \\
(87.5 \%)\end{array}$ & NR & $\begin{array}{l}40 / 40 \\
(100 \%)\end{array}$ & $\begin{array}{l}40 / 40 \\
(100 \%)\end{array}$ & VAS-score & NR & NR & NR & $\begin{array}{l}2.4 \pm 0.6 \\
\text { years }\end{array}$ \\
\hline
\end{tabular}




\begin{tabular}{|c|c|c|c|c|c|c|c|c|c|c|c|}
\hline & $\begin{array}{l}\text { Author, } \\
\text { year }\end{array}$ & $\begin{array}{l}\text { Complete } \\
\text { stone frag- } \\
\text { mentation, } \\
\mathrm{n} / \mathrm{N}(\%)\end{array}$ & $\begin{array}{l}\text { Complete } \\
\text { stone frag- } \\
\text { mentation } \\
\text { after first } \\
\text { session, } \\
\mathrm{n} / \mathrm{N}(\%)\end{array}$ & $\begin{array}{l}\text { Com- } \\
\text { plete } \\
\text { ductal } \\
\text { clear- } \\
\text { ance } \\
\text { after } \\
\text { first } \\
\text { session, } \\
\text { n/N (\%) }\end{array}$ & $\begin{array}{l}\text { Post- } \\
\text { ESWL } \\
\text { ERCP, } \\
\text { n/N (\%) }\end{array}$ & $\begin{array}{l}\text { Com- } \\
\text { plete } \\
\text { ductal } \\
\text { clear- } \\
\text { ance, } \\
\text { n/N (\%) }\end{array}$ & $\begin{array}{l}\text { Method of } \\
\text { pain } \\
\text { measure- } \\
\text { ment }\end{array}$ & $\begin{array}{l}\text { Pain } \\
\text { relief, } \\
\text { n/N (\%) }\end{array}$ & $\begin{array}{l}\text { Com- } \\
\text { plete } \\
\text { pain } \\
\text { relief, } \\
\text { n/N (\%) }\end{array}$ & $\begin{array}{l}\text { Par- } \\
\text { tial } \\
\text { pain } \\
\text { relief, } \\
\mathrm{n} / \mathrm{N} \\
(\%)\end{array}$ & $\begin{array}{l}\text { Follow- } \\
\text { up, m }\end{array}$ \\
\hline 20 & $\begin{array}{l}\text { Ruben- } \\
\text { stein et } \\
\text { al. } 2002 \\
{[15]}\end{array}$ & $\begin{array}{l}23 / 23 \\
(100 \%)\end{array}$ & NR & $\begin{array}{l}68 \% \\
64 \%^{9} \\
71 \%^{10}\end{array}$ & $\begin{array}{l}23 / 23 \\
(100 \%)\end{array}$ & $\begin{array}{l}19 / 23 \\
(82.6 \%) \\
9 / 11 \\
(81.8 \%)^{9} \\
10 / 12 \\
(83.3 \%)^{10}\end{array}$ & NR & NR & NR & NR & NR \\
\hline 21 & $\begin{array}{l}\text { Karasa- } \\
\text { wa et al. } \\
2002 \\
{[26]}\end{array}$ & $\begin{array}{l}3 / 24 \\
(12.5 \%)\end{array}$ & NR & NR & $\begin{array}{l}14 / 24 \\
(58.3 \%)\end{array}$ & $\begin{array}{l}13 / 24 \\
(54.2 \%)\end{array}$ & NR & $\begin{array}{l}11 / 24 \\
(45.8 \%) \\
19 / 20 \\
(95.0 \%)^{8}\end{array}$ & NR & NR & NR \\
\hline 22 & $\begin{array}{l}\text { Brand } \\
\text { et al. } \\
2000 \\
{[24]}\end{array}$ & $\begin{array}{l}29 / 48 \\
(60.4 \%)\end{array}$ & NR & NR & $\begin{array}{l}48 / 48 \\
(100 \%)\end{array}$ & $\begin{array}{l}21 / 48 \\
(43.8 \%)\end{array}$ & $\begin{array}{l}3 \text { types of } \\
\text { pain char- } \\
\text { acteristics }\end{array}$ & $\begin{array}{l}31 / 38 \\
(82 \%)\end{array}$ & $\begin{array}{l}17 / 38 \\
(45 \%)\end{array}$ & $\begin{array}{l}14 / 38 \\
(37 \%)\end{array}$ & $\begin{array}{l}7(5-9)^{2} \\
(n=38)\end{array}$ \\
\hline & $\begin{array}{l}\text { endoscopi } \\
\text { tandard de } \\
\text { y matched } \\
\text { tian with ra } \\
\text { y reported } \\
\text { n with ran } \\
\text { rmediate fo } \\
\text { g-term follc } \\
\text { tal clearan } \\
\text { relief imm } \\
3 \\
\text { coTron } \\
\text { nt Likert-sc } \\
\text { symptom } \\
\text { iiring hospit } \\
\text { nt Likert-sc } \\
\text { ber of hospi } \\
N \text {-up period } \\
\text { ain more th } \\
\text { es of pain c } \\
\text { ds of either } \\
\text { iated with }\end{array}$ & $\begin{array}{l}\text { from } 0-10 \text {. } \\
\text { le= Complete re } \\
\text { f pain. } \\
\text { = Ranging from } \\
\text { lization). } \\
\text { le = Ranging fron } \\
\text { al admissions for } \\
\text { partial clinical su } \\
\text { n times, or wh } \\
\text { aracteristics = Ty } \\
\text { persistent or clus } \\
\text { intensity of }>2\end{array}$ & $\begin{array}{l}\text { f, no pain occur } \\
\text { to } 3 ; 0 \text {, no pain } \\
\text { to } 4 ; 0 \text {, no pai } \\
\text { ain during the } \\
\text { tess, if } 1-5 \text { hos } \\
\text { had undergone } \\
\text { A is characteri } \\
\text { on a visual analo }\end{array}$ & $\begin{array}{l}\text { during the } \\
\text { mild pain ( } \\
\text { 1, mild pain } \\
\text { w-up perio } \\
\text { lizations fo } \\
\text { form of su } \\
\text { as recurre } \\
\text { in for } \geq 2 \text { da } \\
\text { cale (VAS) }\end{array}$ & $\begin{array}{l}\text { low-up peri } \\
\text { use of analg } \\
\text { moderate } \\
\text { compete cl } \\
\text { ain were rec } \\
\text { ry related } t \\
\text { hort episod } \\
\text { week; and ty } \\
\text { l as type C } \mathrm{V}\end{array}$ & $\begin{array}{l}\text { d; partial reli } \\
\text { sics, includin } \\
\text { ain; } 3 \text {, severe } \\
\text { hical success, } \\
\text { rded during } \\
\text { CP during th } \\
\text { s of pain, sep } \\
\text { e } C \text { as an abs } \\
\text { hen }<20 \text { on } V\end{array}$ & $\begin{array}{l}\text { decreased epi } \\
\text { discomfort); } 2 \text {, } \\
\text { ain; } 4 \text {, annihilat } \\
\text { no further hosp } \\
\text { follow-up per } \\
\text { follow-up perio } \\
\text { ated by pain-fr } \\
\text { hce of type A o } \\
\text { (0-100). }\end{array}$ & $\begin{array}{l}\text { des and inte } \\
\text { toderate pai } \\
\text { d; failure, if } \\
\text { e episodes o } \\
\text { type B pain. }\end{array}$ & $\begin{array}{l}\text { requiring a } \\
\text { ity of pain; } \\
\text { tients were } \\
\text { t least mon } \\
\text { in was defir }\end{array}$ & $\begin{array}{l}\text { Igesics); } \\
\text { ad no reli } \\
\text { eded dur } \\
\text { s; type } B \\
\text { d as type }\end{array}$ & $\begin{array}{l}\text { udocyst; } \\
\text { no change } \\
\text { severe pain } \\
\text { ge hospital } \\
\text { prolonged } \\
\text { or B when }\end{array}$ \\
\hline
\end{tabular}

$56.1 \%$ (95\% Cl 42.4-69.4). Stone fragmentation rate was reported in three of the 12 studies with 209 patients, with complete stone fragmentation rate of $86.8 \%(95 \% \mathrm{Cl}$ 61.4-99.5). Post-procedural pancreatitis was described in eight studies with 374 patients and occurred in $3.8 \%(95 \% \mathrm{Cl} 1.8-6.6)$. Cholangitis was described in eight studies with 446 patients and occurred in $1.0 \%(95 \% \mathrm{Cl} 0.19-2.3)$. A second sensitivity analysis was performed after excluding all retrospective studies $(\triangleright \mathrm{Ta}$ ble 1). A total of eight studies with $650(17.1 \%)$ patients were included in the sensitivity analysis. Complete ductal clearance was reached in $63.5 \%$ (95\% Cl 51.7-74.5) of the 636 patients undergoing ESWL. Complete pain relief was described in four of eight prospective studies with 314 patients. The proportion of patients being completely pain free after ESWL was 62.5\% (95\% Cl 48.9-75.1). Stone fragmentation rate was reported in three of eight prospective studies with 285 patients. Complete stone fragmentation rate was $92.7 \%$ (95\% Cl 58.1-97.5). Postprocedural pancreatitis was described in five studies with 418 patients and occurred in $3.1 \%(95 \% \mathrm{Cl} 1.7-5.0)$. Cholangitis was described in six studies with 546 patients and occurred in $0.2 \%(95 \% \mathrm{Cl} 0.00-0.79)$. 


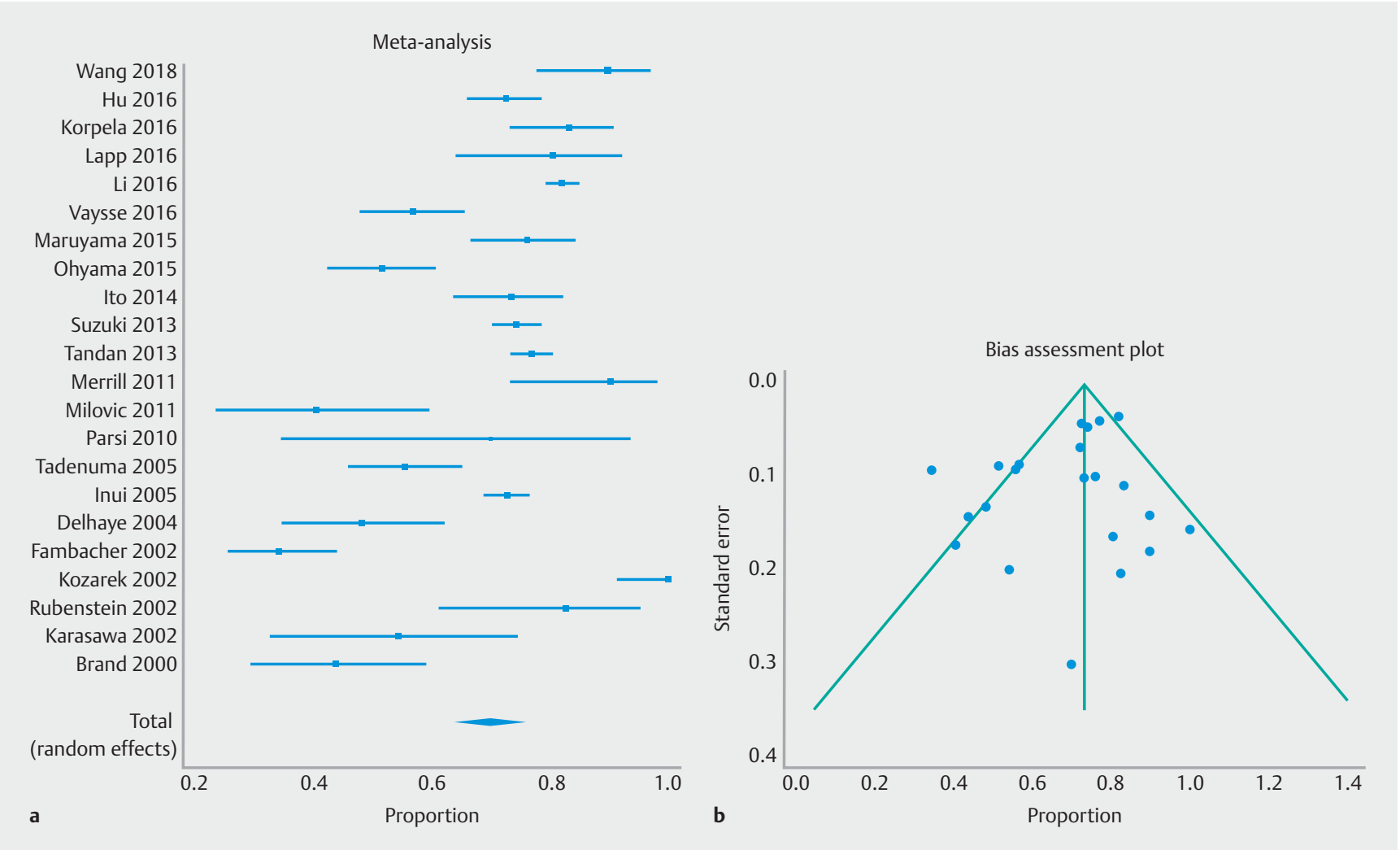

- Fig. 1 a Pooled proportion of complete ductal clearance. b Funnel plot (complete ductal clearance).

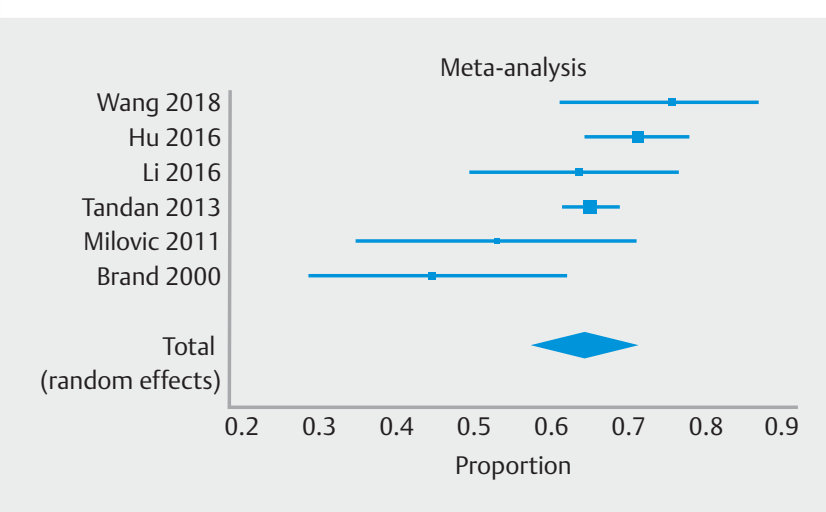

Fig. 2 Pooled proportion of patients being completely pain free after ESWL.

\section{Discussion}

This systematic review and meta-analysis on ESWL for pancreatic duct stones in patients with symptomatic chronic pancreatitis reported a pooled complete ductal clearance of $70 \%$. Complete stone fragmentation was reached in $86 \%$, with $64 \%$ being completely pain free at follow-up. Post-procedural pancreatitis and cholangitis occurred in $4.0 \%$ and $0.5 \%$, respectively, with no mortality reported. Sensitivity analysis only including prospective studies reported similar results, with improvement in complete stone fragmentation rate (93\% versus $86 \%$ ).
Pain is the most frequent symptom in patients with chronic pancreatitis. Currently, a step-up treatment approach is used, including lifestyle modifications and analgesics, endoscopic, and finally surgical treatment $[7,8]$. Treatment goals include pain management, management and prevention of complications (e.g. recurrent flares of cysts), and correction of pancreatic insufficiency.

Conventional endoscopy for the removal of obstructive PD stones with sphincterotomy and basket or balloon extraction, allows for stone extraction in a minority of patients. Conventional endoscopy is regularly limited by factors such as stone size, stone location, and previous gastric surgery [29, 36]. In addition, stone extraction may not be possible if the stone is embedded in the ductal system or when strictures are present [37]. Two retrospective studies reported a $9 \%$ ductal clearance rate after conventional endoscopy in 1041 patients with chronic pancreatitis $[27,29]$, whereas a survey of 125 hospitals reported a $14 \%$ ductal clearance rate in 1834 patients with chronic pancreatitis [38].

As an alternative, initial stone fragmentation by ESWL was introduced [23]. ESWL is indicated for patients with one or multiple radiopaque obstructive intraductal stones larger than 5 $\mathrm{mm}$ located in the head/body of the pancreas, whereas stones that are radiolucent or smaller than $5 \mathrm{~mm}$ should be treated with ERCP only [8]. ESWL is contraindicated in patients with non-correctable coagulation disorders, pregnancy, and presence in the shockwave path of bone, calcified vessels, or lung 
- Table 3 Exocrine and endocrine insufficiency before and after ESWL.

\begin{tabular}{|c|c|c|c|c|c|c|c|}
\hline & \multirow[b]{2}{*}{ Author, year } & \multicolumn{3}{|c|}{ Exocrine insufficiency } & \multicolumn{3}{|c|}{ Endocrine insufficiency } \\
\hline & & Method & Pre-ESWL & Post-ESWL & Method & Pre-ESWL & Post-ESWL \\
\hline 1 & $\begin{array}{l}\text { Wang et al. } \\
2018 \text { [16] }\end{array}$ & NR & NR & NR & NR & NR & NR \\
\hline 2 & $\begin{array}{l}\text { Hu et al. } \\
2016 \text { [19] }\end{array}$ & $\begin{array}{l}\text { Self-reported } \\
\text { steatorrhea }\end{array}$ & $\begin{array}{l}24 / 195 \\
(12.3 \%)\end{array}$ & $34 / 195(17.4 \%)$ & $\begin{array}{l}\text { Self-reported } \\
\text { diabetes }\end{array}$ & $\begin{array}{l}52 / 195 \\
(26.7 \%)\end{array}$ & $57 / 195(29.2 \%)$ \\
\hline 3 & $\begin{array}{l}\text { Korpela et al. } \\
2016 \text { [14] }\end{array}$ & $\begin{array}{l}\text { Fecal elastase in } \\
\text { case of self-re- } \\
\text { ported diarrhea } \\
\text { or steatorrhea }\end{array}$ & $\begin{array}{l}76 \mu g / g \\
(0-608)^{1} \\
(n=25)\end{array}$ & $\begin{array}{l}38 \mu g / g(0-573)^{1} \\
(n=29)\end{array}$ & $\begin{array}{l}\text { Self-reported } \\
\text { diabetes }\end{array}$ & $22 / 83(26.5 \%)$ & $29 / 60(48.3 \%)$ \\
\hline 4 & $\begin{array}{l}\text { Lapp et al. } \\
2016 \text { [31] }\end{array}$ & NR & NR & NR & NR & NR & NR \\
\hline 5 & $\begin{array}{l}\text { Li et al. } 2016 \\
\text { [20] }\end{array}$ & NR & $\begin{array}{l}\text { PPC: } 9 / 55 \\
(16.4 \%)\end{array}$ & PPC: $11 / 55$ (20\%) & $\begin{array}{l}\text { 'Patients with } \\
\text { diabetes follow- } \\
\text { ing ESWL' }\end{array}$ & $\begin{array}{l}\text { PPC: } 10 / 55 \\
(18.2 \%)\end{array}$ & PPC: $11 / 55$ (20.0\%) \\
\hline 6 & $\begin{array}{l}\text { Vaysse et al. } \\
2016 \text { [35] }\end{array}$ & NR & NR & NR & NR & NR & NR \\
\hline 7 & $\begin{array}{l}\text { Maruyama et } \\
\text { al. } 2015 \text { [34] }\end{array}$ & NR & NR & NR & NR & NR & NR \\
\hline 8 & $\begin{array}{l}\text { Ohyama et } \\
\text { al. } 2015 \text { [21] }\end{array}$ & BT-PABA test & $\begin{array}{l}60.7 \pm 18.1 \\
(n=218)\end{array}$ & $60.0 \pm 21.7(n=218)$ & $\begin{array}{l}\text { Definition of } \\
\text { American Dia- } \\
\text { betes Association }\end{array}$ & $\begin{array}{l}28 / 128 \\
(21.9 \%)\end{array}$ & $28 / 128(21.9 \%)$ \\
\hline 9 & $\begin{array}{l}\text { Ito et al. } \\
2014 \text { [30] }\end{array}$ & NR & NR & NR & NR & NR & NR \\
\hline 10 & $\begin{array}{l}\text { Suzuki et al. } \\
2013 \text { [29] }\end{array}$ & NR & NR & NR & NR & NR & NR \\
\hline 11 & $\begin{array}{l}\text { Tandan et al. } \\
2013 \text { [17] }\end{array}$ & $\begin{array}{l}\text { Self-reported } \\
\text { steatorrhea + } \\
\text { required dose of } \\
\text { pancreatic en- } \\
\text { zymes }\end{array}$ & $\begin{array}{l}\text { Intermediate } \\
\text { FU: } 28 / 364 \\
\text { (7.7\%) } \\
\text { Long-term FU: } \\
23 / 272(8.5 \%)\end{array}$ & $\begin{array}{l}\text { Intermediate FU: } \\
\text { 28/364 (7.7\%) } \\
\text { Long-term FU: } \\
23 / 272(8.5 \%)\end{array}$ & $\begin{array}{l}\text { Blood glucose } \\
\text { HbA1c } \\
\text { Required dose of } \\
\text { hypoglycaemic } \\
\text { agents }\end{array}$ & $\begin{array}{l}\text { Intermediate } \\
\text { FU: } 88 / 364 \\
(24.2 \%) \\
\text { Long-term FU: } \\
75 / 272 \\
(27.6 \%)\end{array}$ & $\begin{array}{l}\text { Intermediate FU: } \\
\text { 88/364 (24.2\%) } \\
\text { Long-term FU: } \\
139 / 272(51.1 \%)\end{array}$ \\
\hline 12 & $\begin{array}{l}\text { Merrill et al. } \\
2011 \text { [33] }\end{array}$ & NR & NR & NR & NR & NR & NR \\
\hline 13 & $\begin{array}{l}\text { Milovic et al. } \\
2011 \text { [23] }\end{array}$ & NR & NR & NR & NR & NR & NR \\
\hline 14 & $\begin{array}{l}\text { Parsi et al. } \\
2010 \text { [32] }\end{array}$ & NR & NR & NR & NR & NR & NR \\
\hline 15 & $\begin{array}{l}\text { Tadenuma et } \\
\text { al. } 2005 \text { [22] }\end{array}$ & BT-PABA test & $\begin{array}{l}64.5 \pm 16.1 \\
(n=30)\end{array}$ & $56.2 \pm 12.9(n=30)$ & $\begin{array}{l}\text { Definition of } \\
\text { American Dia- } \\
\text { betes Association }\end{array}$ & $25 / 70(35.7 \%)$ & $40 / 70(57.1 \%)$ \\
\hline 16 & $\begin{array}{l}\text { Inui et al. } \\
2005 \text { [28] }\end{array}$ & BT-PABA test & NR & $\begin{array}{l}3 \text { months FU: } \\
\text { - Improvement: } \\
\text { 488/236 (37.3\%) } \\
\text { - No change: 103/236 } \\
\text { (43.6\%) } \\
\text { - Deterioration: } \\
\text { 45/236 (19.1\%) } \\
1 \text { year FU: } \\
\text { - Improvement: } \\
\text { 65/171 (38.0\%) } \\
\text { - No change: } 49 / 171 \\
\text { (28.7\%) } \\
\text { - Deterioration: } \\
\text { 57/171 (33.3\%) }\end{array}$ & $\begin{array}{l}75-\mathrm{g} \text { oral glucose } \\
\text { tolerance test } \\
\text { Blood glucose } \\
\text { HbA1c } \\
\text { Required dose of } \\
\text { hypoglycaemic } \\
\text { agents }\end{array}$ & NR & $\begin{array}{l}3 \text { months FU: } \\
\text { - Improvement: } \\
\text { 52/233 (22.3\%) } \\
\text { - No change: } 162 / 233 \\
\text { (69.5\%) } \\
\text { - Deterioration: } \\
\text { 19/233 (8.2\%) } \\
\text { 1 year FU: } \\
\text { - Improvement: } \\
\text { 44/181 (24.3\%) } \\
\text { - No change: 85/181 } \\
\text { (47.0\%) } \\
\text { - Deterioration: } 52 / \\
\text { 181 (28.7\%) }\end{array}$ \\
\hline
\end{tabular}


Table 3 (Continuation)

\begin{tabular}{|c|c|c|c|c|c|c|c|}
\hline & \multirow[b]{2}{*}{ Author, year } & \multicolumn{3}{|c|}{ Exocrine insufficiency } & \multicolumn{3}{|c|}{ Endocrine insufficiency } \\
\hline & & Method & Pre-ESWL & Post-ESWL & Method & Pre-ESWL & Post-ESWL \\
\hline 17 & $\begin{array}{l}\text { Delhaye et al. } \\
2004\end{array}$ & $\begin{array}{l}\text { Clinical steator- } \\
\text { rhea that im- } \\
\text { proved with } \\
\text { more than } 1 \\
\text { month of treat- } \\
\text { ment with pan- } \\
\text { creatic enzyme } \\
\text { substitution. }\end{array}$ & $16 / 56(28.6 \%)$ & $34 / 56(60.7 \%)$ & $\begin{array}{l}\text { Oral antidiabtetic } \\
\text { drugs or insulin } \\
\text { use for more than } \\
1 \text { month }\end{array}$ & $16 / 56(28.6 \%)$ & $41 / 56(73.2 \%)$ \\
\hline 18 & $\begin{array}{l}\text { Farnbacher } \\
\text { et al. } 2002 \\
\text { [27] }\end{array}$ & NR & NR & NR & NR & NR & NR \\
\hline 19 & $\begin{array}{l}\text { Kozarek et al. } \\
2002 \text { [18] }\end{array}$ & NR & NR & NR & NR & NR & NR \\
\hline 20 & $\begin{array}{l}\text { Rubenstein } \\
\text { et al. } 2002 \\
{[15]}\end{array}$ & NR & NR & NR & NR & NR & NR \\
\hline 21 & $\begin{array}{l}\text { Karasawa et } \\
\text { al. } 2002 \text { [26] }\end{array}$ & $\begin{array}{l}\text { BT-PABA test } \\
\text { (at admission) } \\
\text { Fecal chymo- } \\
\text { trypsin }\end{array}$ & $\begin{array}{l}74 \pm 22 \% \\
18 \pm 12 \mu \mathrm{U} / \mathrm{g}\end{array}$ & $\begin{array}{l}\text { NR } \\
25 \pm 16 \mu \mathrm{U} / \mathrm{g}\end{array}$ & $\mathrm{HbA1C}$ & $6.6 \pm 2.0 \%$ & $5.4 \pm 0.5 \%$ \\
\hline 22 & $\begin{array}{l}\text { Brand et al. } \\
2000 \text { [24] }\end{array}$ & $\begin{array}{l}\text { Fecal elastase } \\
\text { Fecal chymo- } \\
\text { trypsin } \\
\text { Self-reported } \\
\text { steatorrhea }\end{array}$ & $\begin{array}{l}15 \mathrm{mg} / \mathrm{g} \\
(15-621)^{1} \\
5.4 \mathrm{lU} / \mathrm{g} \\
(0.7-45.6)^{1} \\
13 / 48(27.1 \%)\end{array}$ & $\begin{array}{l}15 \mathrm{mg} / \mathrm{g}(15-619)^{1} \\
6.6 \mathrm{IU} / \mathrm{g}(0.2-47.3)^{1} \\
\text { Improvement in } 10 / \\
13 \text { patients }(77 \%)\end{array}$ & $\begin{array}{l}\text { Fasting blood } \\
\text { glucose } \\
\text { HbA1c } \\
\text { Required dose of } \\
\text { hypoglycemic } \\
\text { agents }\end{array}$ & $32 / 38(84 \%)$ & $27 / 38(71 \%)$ \\
\hline
\end{tabular}

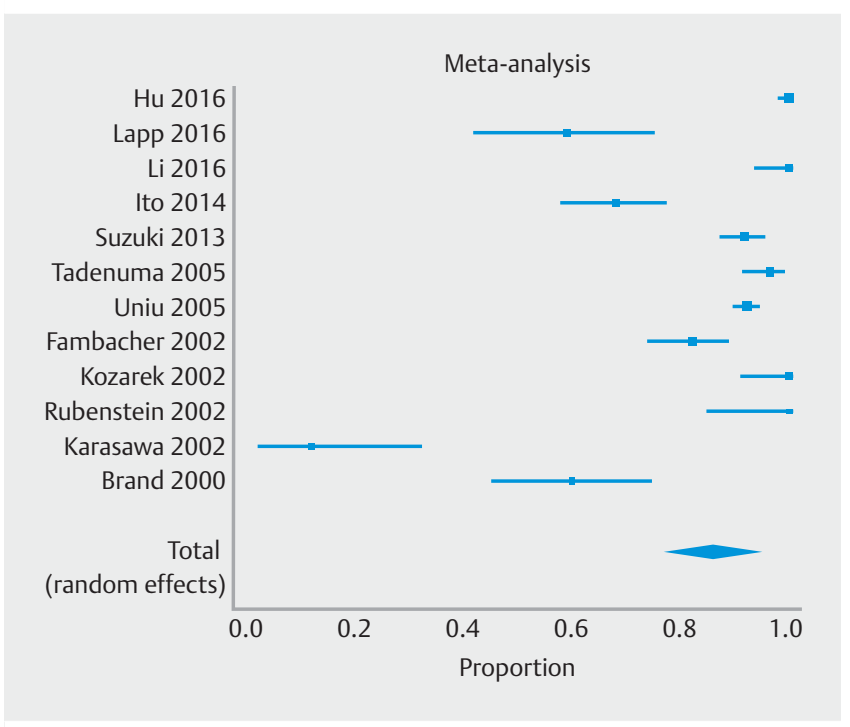

- Fig. 3 Pooled proportion of stone fragmentation. tissue [8]. Specific precautions should be taken for patients with implantable defibrillators and pacemakers [39].

A previous meta-analysis on the efficacy and safety of ESWL reported complete ductal clearance in $70.7 \%$ of the pooled patients comparable to the current study, with complete pain relief in $53 \%$ [10]. Post-procedural pancreatitis was reported in $4.2 \%$, whereas post-procedural cholangitis was not reported. As almost $75 \%$ (20/27) of the included studies was published more than 10 years ago with almost $50 \%$ (13/27) published before the year 2000 , the current study provides an important update on the results of ESWL for PD stones.

Factors associated with more ESWL sessions required for complete stone fragmentation are larger stones [40], multiple stones [41] or stones associated with main pancreatic duct strictures [24]. Multicenter surveys have suggested that stone fragmentation is less frequently successful in low-volume centers, while the type of lithotripsy has been controversial $[29,36,38]$. Factors associated with successful ductal clearance after ESWL are solitary stones [19, 21, 24, 42, 43], stones located in the pancreatic head [19], stones with lower density at CT scan (<820.5 HU) [21], pancreatic stenting prior to ESWL $[14,44]$ and pre-ESWL administration of secretin [44]. 
Endoscopic therapy after ESWL should be restricted to patients with PD strictures or patients with no spontaneous stone clearance after adequate fragmentation with ESWL [8]. The addition of endoscopic therapy to ESWL provided no additional benefit in two studies. Only one randomized controlled trial (RCT) has been published, which showed that combining systematic endoscopy with ESWL adds to the cost of patient care, without improving the outcome of pancreatic pain [11]. This systematic review found similar results.

Intraductal lithotripsy techniques, including laser lithotripsy (LL) and electrohydraulic lithotripsy (EHL), can be attempted either after failure of ESWL or before in an experimental setting. Literature on these lithotripsy techniques in PD stones is scarce, with only one systematic review published showing complete ductal clearance in $43-100 \%$ and adverse events in $0 \%$ to $13.5 \%$ [45]. Large, prospective studies are needed to prove efficiency and safety of these techniques and to compare outcomes with ESWL.

In case of technical or clinical failure of endoscopic therapy, surgical options should be considered [8]. Especially in earlystage chronic pancreatitis, physicians differ in the application of surgical treatment [46]. Currently, multiple RCTs have been published comparing endoscopy with surgery in late-stage chronic pancreatitis. Two recent RCTs on endoscopy versus surgery in late-stage chronic pancreatitis suggested that for pain relief, surgery was superior to endoscopic therapy $[47,48]$. The results of both trials, however, should be interpreted with caution. In the first trial [47], pain relief was reached in $15 \%$ versus $34 \%$ treated with endoscopy versus surgery, respectively, showing that neither of these treatment options was entirely satisfactory. The applied endoscopic therapy was suboptimal, as neither ESWL nor cumulative stenting were used. In addition, different surgical procedures were performed [47]. In the second trial, only 39 patients were included; all of them had advanced chronic pancreatitis and most were opioid-dependent [48]. In both trials, only patients with late-stage chronic pancreatitis were included, and therefore the results of the trials cannot been extrapolated to all patients with chronic pancreatitis, especially not to patients with early-stage chronic pancreatitis.

Therefore, the timing of surgical intervention remains matter of debate [49]. Whether early surgery for chronic pancreatitis improves pain control and pancreatic function compared with the current step-up practice should be evaluated in a large multicenter RCT and validated afterwards. A recently published multicenter RCT showed that early surgery compared with an endoscopy-first approach resulted in lower pain scores when integrated over 18 months. In addition, the total number of interventions was lower in the early surgery group (median, 1 vs 3; $P<.001$ ), altough complications, mortality, pancreatic function and quality of life were comparable [50]. However, further research is needed to assess persistence of differences over time and to replicate the study findings. Despite these results, early surgery strategy may not be easily generalized as most patients and their refering physicians would prefer a non-invasive procedure as first step management.
This study has several limitations. First, each of the included studies were observational with several retrospective studies, potentially leading to selection bias. Furthermore, multiple outcomes were heterogeneously defined among the included studies. For example, meta-analysis on complete ductal clearance revealed an $\mathrm{I}^{2}$ of $92.6 \%$. Variation between studies regarding this outcome might be caused by heterogeneity in study protocol, for example, rather than by chance alone. Therefore, the results of the meta-analysis should be interpreted with caution. Furthermore, due to heterogeneity, improvement in quality of life and in endocrine and exocrine insufficiency could not be quantified. In addition, there was a lack of objective means of determining 'complete ductal clearance' and 'complete stone fragmentation', which explains the significant heterogeneity $\left(I^{2}>90 \%\right)$ among the studies. Furthermore, no consensus existed amongst the studies in the grading of gastrointestinal procedure-related complications. As a result, we chose to only report post-procedural pancreatitis and cholangitis. Furthermore, studies were performed in different countries with different patient demographics, various etiologies for chronic pancreatitis and stone formation. Baseline characteristics were not available for all the included studies, and therefore no comparison could be performed. Finally, indications of endoscopic therapy following ESWL were not clearly described in multiple studies. The strength of this study is the extensive, detailed overview of the effects of ESWL for PD stones regarding multiple outcomes and of the heterogeneity that exists in current literature.

\section{Conclusion}

This meta-analysis suggests that treatment with ESWL results in complete ductal clearance in $70 \%$ of patients, resulting in absence of pain in over half of patients with symptomatic chronic pancreatitis caused by obstructing pancreatic duct stones. Therefore, ESWL should still be adhered to as a first alternative to conventional endoscopic techniques as long as there are no convincing data about EHL or laser lithotripsy. The available literature, however, is heterogeneous and therefore pragmatic multicenter RCTs in experienced centers are required. In addition, the application of $\mathrm{LL}$ and $\mathrm{EHL}$ as alternative techniques should be further explored in larger, prospective studies.

\section{Competing interests}

The authors declare that they have no conflict of interest.

\section{References}

[1] Yadav D, Timmons L, Benson JT et al. Incidence, prevalence, and survival of chronic pancreatitis: a population-based study. Am J Gastroenterol 2011; 106: 2192-2199

[2] Frulloni L, Gabbrielli A, Pezzilli R et al. Chronic pancreatitis: report from a multicenter Italian survey (PanCrolnfAISP) on 893 patients. Dig Liver Dis 2009; 41: 311-317 
[3] Schneider A, Lohr JM, Singer MV. The M-ANNHEIM classification of chronic pancreatitis: introduction of a unifying classification system based on a review of previous classifications of the disease. J Gastroenterol 2007; 42: 101-119

[4] Mullady DK, Yadav D, Amann ST et al. Type of pain, pain-associated complications, quality of life, disability and resource utilisation in chronic pancreatitis: a prospective cohort study. Gut 2011; 60: 77-84

[5] White TT, Bourde J. A new observation on human intraductal pancreatic pressure. Surg Gynecol Obstet 1970; 130: 275-278

[6] Drewes AM, Krarup AL, Detlefsen S et al. Pain in chronic pancreatitis: the role of neuropathic pain mechanisms. Gut 2008; 57: 1616-1627

[7] Drewes AM, Bouwense SAW, Campbell CM et al. Guidelines for the understanding and management of pain in chronic pancreatitis. Pancreatology 2017; 17: 720-731

[8] Dumonceau JM, Delhaye M, Tringali A et al. Endoscopic treatment of chronic pancreatitis: European Society of Gastrointestinal Endoscopy (ESGE) Guideline - Updated August 2018. Endoscopy 2019; 51: 179193

[9] Lohr JM, Dominguez-Munoz E, Rosendahl J et al. United European Gastroenterology evidence-based guidelines for the diagnosis and therapy of chronic pancreatitis (HaPanEU). United European Gastroenterol J 2017; 5: 153-199

[10] Moole H, Jaeger A, Bechtold ML et al. Success of Extracorporeal shock wave lithotripsy in chronic calcific pancreatitis management: a metaanalysis and systematic review. Pancreas 2016; 45: 651-658

[11] Dumonceau JM, Costamagna G, Tringali A et al. Treatment for painful calcified chronic pancreatitis: extracorporeal shock wave lithotripsy versus endoscopic treatment: a randomised controlled trial. Gut 2007; 56: 545-552

[12] Wells GASB, O'Connell D et al. The Newcastle-Ottawa Scale (NOS) for assessing the quality of nonrandomised studies in meta-analyses. 2000: Available from: http://www.ohri.ca/programs/clinical_epidemiology/oxford.asp

[13] Higgins JP, Thompson SG, Deeks J] et al. Measuring inconsistency in meta-analyses. BMJ (Clinical research ed) 2003; 327: 557-560

[14] Korpela T, Udd M, Tenca A et al. Long-term results of combined ESWL and ERCP treatment of chronic calcific pancreatitis. Scand J Gastroenterol 2016; 51: 866-871

[15] Rubenstein JN, Parsons WG, Kim SC et al. Extracorporeal shock wave lithotripsy of pancreatic duct stones using the Healthtronics LithoTron lithotriptor and the Dornier HM3 lithotripsy machine. J Urol 2002; 167: 485-487

[16] Wang D, Ji JT, Xin L et al. Extracorporeal shock wave lithotripsy for chronic pancreatitis patients with stones after pancreatic surgery. Pancreas 2018; 47: 609-616

[17] Tandan M, Reddy DN, Talukdar R et al. Long-term clinical outcomes of extracorporeal shockwave lithotripsy in painful chronic calcific pancreatitis. Gastrointest Endosc 2013; 78: 726-733

[18] Kozarek RA, Brandabur JJ, Ball TJ et al. Clinical outcomes in patients who undergo extracorporeal shock wave lithotripsy for chronic calcific pancreatitis. Gastrointest Endosc 2002; 56: 496-500

[19] Hu LH, Ye B, Yang YG et al. Extracorporeal shock wave lithotripsy for chinese patients with pancreatic stones: a prospective study of 214 cases. Pancreas 2016; 45: 298-305

[20] Li BR, Liao Z, Du TT et al. Extracorporeal shock wave lithotripsy is a safe and effective treatment for pancreatic stones coexisting with pancreatic pseudocysts. Gastrointest Endosc 2016; 84: 69-78

[21] Ohyama H, Mikata R, Ishihara T et al. Efficacy of stone density on noncontrast computed tomography in predicting the outcome of extracorporeal shock wave lithotripsy for patients with pancreatic stones. Pancreas 2015; 44: 422-428
[22] Tadenuma H, Ishihara T, Yamaguchi T et al. Long-term results of extracorporeal shockwave lithotripsy and endoscopic therapy for pancreatic stones. Clin Gastroenterol Hepatol 2005; 3: 1128-1135

[23] Milovic V, Wehrmann T, Dietrich CF et al. Extracorporeal shock wave lithotripsy with a transportable mini-lithotripter and subsequent endoscopic treatment improves clinical outcome in obstructive calcific chronic pancreatitis. Gastrointest Endosc 2011; 74: 1294-1299

[24] Brand B, Kahl M, Sidhu S et al. Prospective evaluation of morphology, function, and quality of life after extracorporeal shockwave lithotripsy and endoscopic treatment of chronic calcific pancreatitis. Am J Gastroenterol 2000; 95: 3428-3438

[25] Delhaye M, Arvanitakis M, Verset G et al. Long-term clinical outcome after endoscopic pancreatic ductal drainage for patients with painful chronic pancreatitis. Clin Gastroenterol Hepatol 2004; 2: 1096-1106

[26] Karasawa Y, Kawa S, Aoki Y et al. Extracorporeal shock wave lithotripsy of pancreatic duct stones and patient factors related to stone disintegration. J Gastroenterol 2002; 37: 369-375

[27] Farnbacher MJ, Schoen C, Rabenstein T et al. Pancreatic duct stones in chronic pancreatitis: criteria for treatment intensity and success. Gastrointest Endosc 2002; 56: 501-506

[28] Inui K, Tazuma S, Yamaguchi T et al. Treatment of pancreatic stones with extracorporeal shock wave lithotripsy: results of a multicenter survey. Pancreas 2005; 30: 26-30

[29] Suzuki Y, Sugiyama M, Inui K et al. Management for pancreatolithiasis: a Japanese multicenter study. Pancreas 2013; 42: 584-588

[30] Ito K, Igarashi $\mathrm{Y}$, Okano $\mathrm{N}$ et al. Efficacy of combined endoscopic lithotomy and extracorporeal shock wave lithotripsy, and additional electrohydraulic lithotripsy using the SpyGlass direct visualization system or X-ray guided EHL as needed, for pancreatic lithiasis. Biomed Res Int 2014; 2014: 732781

[31] Lapp RT, Wolf JS Jr. et al. Duct diameter and size of stones predict successful extracorporeal shock wave lithotripsy and endoscopic clearance in patients with chronic pancreatitis and pancreaticolithiasis. Pancreas 2016; 45: 1208-1211

[32] Parsi MA, Stevens T, Lopez R et al. Extracorporeal shock wave lithotripsy for prevention of recurrent pancreatitis caused by obstructive pancreatic stones. Pancreas 2010; 39: 153-155

[33] Merrill JT, Mullady DK, Early DS et al. Timing of endoscopy after extracorporeal shock wave lithotripsy for chronic pancreatitis. Pancreas 2011; 40: 1087-1090

[34] Maruyama M, Watanabe T, Kanai K et al. Extracorporeal shock wave lithotripsy treatment of pancreatic stones complicated with advanced stage autoimmune pancreatitis. BMC Gastroenterol 2015; 15: 28

[35] Vaysse T, Boytchev I, Antoni G et al. Efficacy and safety of extracorporeal shock wave lithotripsy for chronic pancreatitis. Scand J Gastroenterol 2016; 51: 1380-1385

[36] Sherman S, Lehman GA, Hawes RH et al. Pancreatic ductal stones: frequency of successful endoscopic removal and improvement in symptoms. Gastrointest Endosc 1991; 37: 511-517

[37] Delhaye M, Vandermeeren A, Baize M et al. Extracorporeal shockwave lithotripsy of pancreatic calculi. Gastroenterology 1992; 102: $610-620$

[38] Inui K, Masamune A, Igarashi Y et al. Management of pancreatolithiasis: a nationwide survey in Japan. Pancreas 2018; 47: 708-714

[39] Crossley GH, Poole JE, Rozner MA et al. The Heart Rhythm Society (HRS)/American Society of Anesthesiologists (ASA) Expert Consensus Statement on the perioperative management of patients with implantable defibrillators, pacemakers and arrhythmia monitors: facilities and patient management: executive summary this document was developed as a joint project with the American Society of Anesthesiologists (ASA), and in collaboration with the American Heart Association (AHA), and the Society of Thoracic Surgeons (STS). Heart Rhythm 2011; 8: e1-e18 
[40] Nguyen-Tang T, Dumonceau JM. Endoscopic treatment in chronic pancreatitis, timing, duration and type of intervention. Best Pract Res Clin Gastroenterol 2010; 24: 281-298

[41] Tandan M, Reddy DN, Santosh D et al. Extracorporeal shock wave lithotripsy and endotherapy for pancreatic calculi-a large single center experience. Indian J Gastroenterol 2010; 29: 143-148

[42] Adamek HE, Jakobs R, Buttmann A et al. Long term follow up of patients with chronic pancreatitis and pancreatic stones treated with extracorporeal shock wave lithotripsy. Gut 1999; 45: 402-405

[43] Dumonceau JM, Deviere J, Le Moine O et al. Endoscopic pancreatic drainage in chronic pancreatitis associated with ductal stones: longterm results. Gastrointest Endosc 1996; 43: 547-555

[44] Choi EK, McHenry L, Watkins JL et al. Use of intravenous secretin during extracorporeal shock wave lithotripsy to facilitate endoscopic clearance of pancreatic duct stones. Pancreatology 2012; 12: 272275

[45] Beyna T, Neuhaus H, Gerges C. Endoscopic treatment of pancreatic duct stones under direct vision: Revolution or resignation? Systematic review Dig Endosc 2018; 30: 29-37
[46] Lamme B, Boermeester MA, Straatsburg IH et al. Early versus late surgical drainage for obstructive pancreatitis in an experimental model. Br J Surg 2007; 94: 849-854

[47] Dite P, Ruzicka M, Zboril V et al. A prospective, randomized trial comparing endoscopic and surgical therapy for chronic pancreatitis. Endoscopy 2003; 35: 553-558

[48] Cahen DL, Gouma DJ, Nio Y et al. Endoscopic versus surgical drainage of the pancreatic duct in chronic pancreatitis. N Engl J Med 2007; 356: 676-684

[49] Drewes AM, Kempeneers MA, Andersen DK et al. Controversies on the endoscopic and surgical management of pain in patients with chronic pancreatitis: pros and cons! Gut 2019: doi:10.1136/gutjnl-2019318742

[50] Issa Y, Kempeneers MA, Bruno MJ et al. Effect of early surgery vs endoscopy-first approach on pain in patients with chronic pancreatitis: the ESCAPE randomized clinical trial. JAMA 2020; 323: 237-247 